\title{
Review Article \\ The Problem of Antigen Affinity Discrimination in B-Cell Immunology
}

\author{
Subhadip Raychaudhuri ${ }^{1,2}$ \\ ${ }^{1}$ Department of Chemistry, University of California Davis, Davis, CA 95616, USA \\ ${ }^{2}$ Indraprastha Institute of Information Technology, Delhi 110020, India \\ Correspondence should be addressed to Subhadip Raychaudhuri; raychaudhuri@ucdavis.edu
}

Received 4 February 2013; Accepted 26 February 2013

Academic Editors: H. Kono and J. R. C. Piqueira

Copyright (c) 2013 Subhadip Raychaudhuri. This is an open access article distributed under the Creative Commons Attribution License, which permits unrestricted use, distribution, and reproduction in any medium, provided the original work is properly cited.

$\mathrm{B}$ and T lymphocytes activate the humoral and cellular arms of the adaptive immune system. The adaptive strategy works because receptors of adaptive immune cells can mount an immune response based on their affinity for antigens. Thus, affinity discrimination is central to adaptive immunity and has important biomedical ramifications. Due to its intricate connection to the affinity maturation process, affinity discrimination has a special significance in B-cell-mediated immune response. The role of affinitymatured high-affinity antibodies is increasingly recognized in vaccine development. In this paper, we discuss the recent progress made in mathematical and computational studies to explore the cellular and molecular mechanisms of B-cell affinity discrimination. Formation of B-cell receptor (BCR) oligomers and BCR-lipid rafts, upon antigenic stimulation, emerge to be key factors in B-cell affinity discrimination (at the level of single cells). It also provides a new way of thinking about kinetic proofreading and serial triggering, concepts that have been widely utilized to understand affinity discrimination in adaptive immune cells. Potential future applications of mathematical and computational modeling of affinity discrimination are discussed in the context of autoimmune disorders and vaccine design.

\section{Introduction}

The adaptive immune system confers an extraordinary protection to higher organisms from invading foreign pathogens. The adaptive feature of the immune system relies on clonal expansion of B and T lymphocytes (upon antigenic stimulation) equipped with high-affinity antigen-specific receptors and generation of memory cells with similar high-affinity receptors (consistent with the clonal selection theory [1]), while a large pool of lower affinity receptors are maintained for efficient sampling of unforeseen antigenic epitopes. Clearly, such a strategy works because receptors of adaptive immune cells can mount an immune response based on recognition of antigenic affinity (affinity discrimination). In B cells, the additional flexibility of generating higher affinity receptors through the genetic process of somatic hypermutation aids selective expansion of high-affinity clones (also known as affinity maturation), making it a powerful strategy to combat foreign pathogens. Thus, in B cells, affinity discrimination and affinity maturation are intricately linked. In addition to its fundamental importance in the mechanism of immune cell activation, the role of affinity-matured antibodies is now increasingly explored in vaccine development [2$6]$.

Antigenic affinity discrimination, by adaptive immune cells, is key to understanding infectious and autoimmune diseases and developing effective preventive measures. However, the mechanisms of affinity discrimination are not clearly elucidated and attracting much recent attention [7-16]. Mathematical and computational modeling has long been utilized to provide mechanistic insight into complex mechanisms of the immune system and emerged as a powerful tool to solve immunological problems $[17,18]$. In this paper, we review the recent mathematical and computational efforts to elucidate the mechanisms of antigen affinity discrimination (by lymphocytes of the adaptive immune system). Experimental data on affinity discrimination and affinity maturation has infused considerable excitement in the field [7-10, 12-14, 19-21]. Recent advent of experimental technologies, such as powerful imaging methods and single-cell approaches, will presumably 
generate new data at a spatiotemporal resolution that has not been achieved before. On the theoretical side, highly complex mathematical and computational models of biological systems will become increasingly possible to develop and solve (utilizing novel approaches and increasingly available computing power).

\section{The Problem of Affinity Discrimination}

Affinity discrimination has a central role in immune activation and adaptation. In the simplest manner, the immune system has evolved to achieve the following tasks:

(1) discern nonself-antigens from self-antigens and mount an immune response only against those foreign pathogens;

(2) adapt to the changing pathogenic environment and keep immunological memory; this type of immunity allows generation of variable amount of response to the same nonself-antigen (e.g., repeated exposure of the same antigen is interpreted as increased degree of "nonselfness").

Cells of both innate and adaptive immunity work in concert to mount a successful immune response against harmful foreign pathogens. Immune receptors tend to bind to foreign proteins (or peptides) with sufficiently high affinity leading to robust intracellular signaling and activation, while the affinity for self-antigens typically falls below the threshold of activation (leading to self-/nonself-discrimination). Affinity measures the strength with which a cell surface receptor binds a ligand molecule at a single site.

Adaptive immune cells (starting in vertebrates), interestingly, can discriminate antigen affinity by generating variable amount of immune response depending on the affinity for foreign antigen ligands. This kind of affinity discrimination allows the immune system to respond in an adaptive manner: (i) by adjusting the immune response to a changing pathogenic load (such as through affinity maturation) and (ii) by generating and maintaining high-affinity clones of receptors (such as in memory cells). Such a complex task is achieved because cells of the adaptive immune system, such as B and T lymphocytes, are specific to a foreign antigen, and usually only a few copies (1-10) of such specific lymphocytes are present. At the same time, a huge repertoire of antigenspecific lymphocytes is present for efficient coverage of the antigenic epitope space.

Mathematical and computational modeling as used in elucidating various aspects of affinity discrimination is the focus of this paper. Affinity discrimination by both $B$ and $\mathrm{T}$ lymphocytes is discussed. We primarily focus on affinity discrimination in B lymphocytes because of its critical role in the affinity maturation process as well as in vaccine design. In addition, recent experimental and theoretical efforts seem to elucidate the mechanism of B-cell affinity discrimination at a detailed molecular level.

2.1. Affinity Discrimination and Functional Outcomes of Immune Cell Activation. Affinity discrimination is intricately

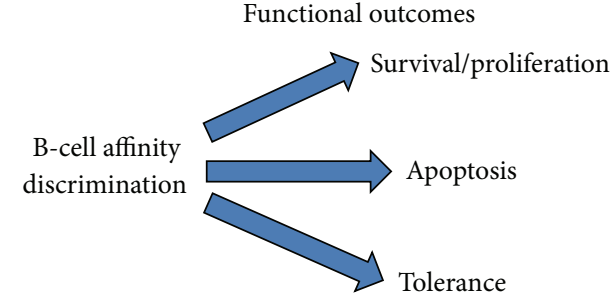

FIGURE 1: Schematic showing that B-cell affinity discrimination is linked to fundamental outcomes of B-cell activation.

linked to the functional outcomes (such as survival/proliferation, apoptosis, and tolerance) and/or phenotypes of immune cell activation (Figure 1) [22-24].

In B cells, affinity discrimination is also important in late stage lineage commitment such as between plasma cell and memory cell generation $[23,24]$. These types of problems in immunology, where affinity for antigens determines the functional outcomes of immune cell activation, can be considered as an important class of affinity discrimination problem. Two examples are provided as follows.

(1) Maturation of lymphocytes (from a large pool of immature cells) is one of those problems that can be considered as an affinity discrimination problem. Here, antigen affinity is the key determinant of functional outcomes of immune cell activation. In negative selection, strong binding to self-antigens (above a threshold affinity value) results in apoptotic cell death for both B and T lymphocytes. Very weak binding to self-antigens is known to deprive immature immune cells of the essential survival signal and also results in activation of cell death pathways. This affinitydependent selection and maturation process confers protection from autoimmunity.

(2) Cytotoxic response of the CD8 T cells is known to be regulated by antigen binding affinity where only the agonist ligands (that bind TCRs with sufficiently high affinity) generate strongest response. Weak agonists (having lower affinity TCRs or very high TCRpMHC bond half-life) are able to generate only partial response. One partial agonist was shown to activate only the Fas (CD95) receptors but not the perforin/granzyme-mediated cytotoxicity [25]. Clearly, the affinity-dependent response of cytotoxic $\mathrm{T}$ cells is a key to cellular immunity against infections and tumor and should have important ramifications in cancer immunotherapy.

2.2. Affinity Discrimination and Affinity Maturation. B lymphocytes have an ability to adapt to the changing pathogenic load by continuously generating diversity in the variable region of the receptor (somatic hypermutation leading to variations in antigen binding affinity) and selecting the highaffinity clones, a feature known as affinity maturation. It is a strategy by which B lymphocytes optimize the immune 
response given a pathogenic load. In B cells, affinity discrimination implies increasing level of signaling response as the affinity for antigen is increased; thus, such a monotonic response imparts advantage to high-affinity receptors clonally selected by affinity maturation. B cells equipped with such high-affinity receptors are able to mount stronger signaling response (such as cytokine production). Effector functions are also enhanced due to secretion of higher affinity antibodies that are more efficient in eliminating antigens. In addition, effector functions can be enhanced by other factors, such as by higher degree of IgM polymerization (of secreted antibodies) with increased affinity of antigens as observed recently $[14,21,26]$. Earlier studies indicated factors such as (i) competition for antigens, (ii) variability in T-cell interaction, and (iii) differential activation of apoptosis (cell death) that could lead to selection of high-affinity receptors during the process of affinity maturation [27]. More recent observations, however, emphasize the role of antigen affinity discrimination at the level of single-cell activation $[12,13,15,16]$. It is expected that insights gained from such studies will be increasingly used in understanding infectious diseases and autoimmune disorders as well as in design of vaccines.

\section{Understanding Affinity Discrimination: Kinetic Proofreading and Serial Triggering}

How do adaptive immune cells, such as B lymphocytes, respond to varying affinity of antigens defining the biological problem of affinity discrimination? Adaptive immune cells are equipped with immune receptors that can recognize antigens and sense the antigen affinity using a membrane proximal signaling network. Phosphorylation of the signaling chains (ITAMs) of those immune receptors can be taken to be a measure of early signaling that is propagated downstream (intracellular signaling) by the coordinated action of signaling kinases and phosphatases finally leading to gene transcription and cellular response. How does the affinity affect the membrane proximal and further downstream signaling activation? The very first event of immune recognition, namely, receptor-antigen binding, should increase with increasing affinity. Such a result makes sense but can also be demonstrated by the following set of kinetic rate equations [28] for receptor- $(R-)$ ligand $(L)$ binding $R+L \rightleftarrows \mathrm{C}$ :

$$
\frac{d C}{d t}=-\frac{d R}{d t}=-\frac{d L}{d t}=k_{\mathrm{on}} R L-k_{\mathrm{off}} C .
$$

The number of complex formed $C(t)$ (also its final steadystate value) should increase with increasing affinity $K_{A}=$ $k_{\text {on }} / k_{\text {off }}$. One can also include diffusion into the above rate equations by incorporating $D \nabla^{2} C$-type terms and changing the equations to PDEs. The number of receptor-ligand bonds formed still increases with increasing affinity. However, it is not obvious that signaling through the receptor-ligand bonds will also increase with increasing affinity. Low affinity antigens have the ability to quickly dissociate from an immune cell receptor, which may lead to serial activation of a large number of such receptors in a small amount of time (an effect known as "serial triggering") resulting in decrease in signaling with increasing affinity. Such a result can also be demonstrated by carrying out Monte Carlo simulations of receptor-ligand bond formation and signaling [29, 30]. The following reaction moves need to be included in the simulation: (i) binding/unbinding of receptor-ligand bonds and (ii) conversion of receptors to a signaling able conformation by a signaling kinase. One needs to assume that signaling kinases can induce a conformational change (to a signaling active form) in the immune receptor only when they are bound to antigens. Experimental studies of immune cell receptors, however, do not indicate monotonically decreasing signaling behavior as the affinity for antigen is increased. In $\mathrm{T}$ cells, some studies indicate a nonmonotonic signaling behavior with increasing affinity $[10,11]$. In B cells, interestingly, the signaling can only increase (monotonic) with increased affinity [7, 8, 12, 13], a behavior also known as graded response. Clearly, the molecular mechanism of affinity discrimination in adaptive immune cells needs to be addressed. The problem of affinity discrimination should be relevant for any immune cells possessing MIRR (multichain immune recognition receptor) family of receptors, especially in B and T lymphocytes. Initial studies in T cells utilized the concepts of kinetic proofreading [31] and serial triggering [32, 33] to explain the observed nonmonotonic behavior in signaling response $[10,11]$.

The idea of kinetic proofreading was applied to understand the extraordinary specificity of T-cell receptors (TCRs) in antigen recognition [31]. It was thought that TCR molecules had to undergo, upon antigen binding, a series of conformational changes before they can participate in downstream signaling activation. If antigen detached (from the TCR) before completion of all the necessary conformational changes, the TCR would revert to its basal inactive form. Such a mechanism would favor high-affinity receptors to reach the signaling capable conformation as they are typically bound to antigens for a longer period of time. In addition, a kinetic proofreading-based mechanism would generate a sharp threshold for affinity only above which it will be possible to complete the required series of conformation changes (leading to specific recognition). In the original proposal of kinetic proofreading, a simple ODE-based model was used to elucidate the above mechanisms in a quantitative manner [31].

The ability of single antigens to serially activate many immune cell receptors, as mentioned earlier, decreases with increasing affinity. This effect, known as serial triggering, provides signaling advantage to low-affinity binding. Therefore, the effect of serial triggering, on affinity discrimination, is opposite to that of kinetic proofreading. Serial engagement and serial triggering (of many receptors by a single antigen) are a natural outcome of binding/unbinding of receptorligand pairs and diffusive transport of individual molecules. Serial triggering was initially demonstrated in the context of T-cell activation by showing that a low number of antigens could lead to internalization of many more TCRs [32]. Though it was not a direct observation of serial activation of many TCRs by a single antigen and one can argue that some TCRs got internalized by other mechanisms [34]. However, it seems reasonable to assume that a single antigen 
can serially bind and activate many TCRs. Later theoretical studies estimated serial engagement of TCRs using a mathematical model of diffusion and binding [35]. In this context, exploration of the effect of coagonist activity of lowaffinity self-peptides (in presence of agonist peptides) might be insightful [36-38].

Initial studies in $\mathrm{T}$ cells demonstrated that a competition between kinetic proofreading and serial triggering could lead to an optimal affinity value $\left(K_{A}=k_{\text {on }} / k_{\text {off }}\right)$ for which the activation is maximal ([10] and references therein, [11]). The dissociation rate $k_{\text {off }}$ emerged as a key parameter, as maximal signaling (in strong agonists) was thought to arise from an optimal half-life of TCR-pMHC binding (that is sufficient for kinetic proofreading yet allows substantial serial triggering). A PDE-based mathematical model was proposed to capture the competing effect of kinetic proofreading and serial triggering [11]. Serial triggering emerges naturally in such a model of diffusion and binding/unbinding reactions (between TCRs and antigens). Kinetic proofreading in the model originated from the requirement that TCRs need to undergo a series of conformational changes before they reach a signaling active conformation. A TCR dimerization model was also considered in this study; however, it was not essential to achieve the nonmonotonic affinity discrimination. It was also assumed that TCRs remained in a signaling active state (capable of internalization) even after they detached from antigen but if already completed the required sequence of conformational changes. The ability of signaling active TCRs to retain the ability to get degraded was shown to be necessary for the nonmonotonic affinity dependence observed in TCR downregulation. Some of the later experimental studies questioned the observation of nonmonotonic affinity discrimination in T cells; instead, an affinity-dependent increase in signaling was proposed [39, 40]. To address these two disparate types of affinity discrimination in T cells, a mathematical/computational model based on kinetic proofreading with rebinding events was proposed $[41,42]$. Whether reduced diffusion of receptors (or antigens), such as that would occur in a lipid raft (or a TCR microcluster), can affect the rebinding events needs to be explored.

In an attempt to explore the affinity discrimination in $\mathrm{B}$ cells (which is different from affinity discrimination in $\mathrm{T}$ cells even at a qualitative level), a Monte Carlo model of B-cell membrane proximal signaling was developed [15]. In this model, kinetic proofreading was captured in an effective manner by demanding that B-cell receptors need to stay bound to antigens for a given period of time before they are signaling capable. This threshold time, which can be treated as a simulation parameter, was varied in controlled in silico experiments. In order to achieve the monotonically increasing (graded) signaling with increasing affinity, as observed in B-cell affinity discrimination experiments, a significant kinetic proofreading time ( 10 seconds) was needed so that it could overcome the competing loss in serial triggering across all physiological affinity values [15]. A key limitation of this model is that it cannot account how signaling kinase Lyn (Src family kinase) access B-cell receptors before it phosphorylates the signaling chains of BCRs.
Whether kinetic proofreading is introduced through an effective threshold time (as in [15]), or by demanding a series of conformational changes that immune receptors need to complete (as in [11]), these models do not associate kinetic proofreading with any physical events at the molecular level. With the advent of modern experimental techniques, however, molecular level details of immune recognition and signaling activation are increasingly explored. The concepts such as kinetic proofreading and serial triggering need to address these more recent experimental findings. In addition, any theoretical model of affinity discrimination (in immune cells) should be able to analyze recent data generated by experimental labs. We will later see that both kinetic proofreading and serial triggering can emerge naturally in a more molecular level model of affinity discrimination (such as in a lipid raft model). Interestingly, kinetic proofreading requirement becomes affinity dependent in such models, providing completely new insight into the problem of affinity discrimination. Before we describe such molecular level elucidations in mathematical and computational models, we need to discuss experimental studies on affinity discrimination including some of the more recent findings.

\section{Experimental Studies of Affinity Discrimination}

In the first set of experiments, a variation in the BCR affinity for antigen resulted in differential downstream signaling response, such as expression of cytokines, cell surface activation markers, antibody $(\mathrm{Ab})$ generation, and commitment to distinct differentiation pathways [7, 8, 19, 20, 22-24]. In these experiments, downstream signaling events showed more pronounced activation with increased affinity of antigens. In addition, it has recently been shown that increased strength of BCR-antigen interaction enhances the degree of IgM polymerization through posttranslational modifications [14, $21,26]$. Thus, production of B cells with high-affinity receptors, through somatic hypermutation and clonal selection, provides a unique advantage to B cells to mount increasingly stronger immune response and differentially regulate B-cell fate. However, one may ask how does this affinity-dependent B-cell response (and functional outcomes) originate from antigen recognition-induced earliest membrane proximal signaling events?

Biochemical and genetic studies, carried out in the past, have characterized the downstream signaling pathways that connect the phosphorylated BCR $\operatorname{Ig} \alpha$ and $\operatorname{Ig} \beta$ chains to Bcell activation [43-47]. However, the initial molecular events (subsequent to BCR-antigen binding) that lead to membrane proximal signaling activation are not well understood [4851]. A striking feature of more recent experimental studies on immune cell activation is that those studies started to elucidate the spatial events, such as molecular clustering, during the earliest phases of immune cell activation.

Recent in vitro experiments showed that initial engagement of antigens leads to formation of BCR microclusters resulting in a spreading and contraction response (of Bcell membranes) in an affinity-dependent manner (enhanced response with increasing affinity) [12]. The signaling basis 
of such affinity-dependent spreading of B cells, however, remained poorly understood. Batista and coworkers have emphasized the role of the cytoskeleton in the initial formation of BCR microclusters and subsequent regulation of B-cell activation [50, 52-54]. Cytoskeletal elements are intricately linked to the plasma membrane and modulate diffusion of lipids and proteins in the cell membrane. Whether actin cytoskeleton induces an initial BCR microclustering or it simply undergoes rearrangement to assist the already forming BCR clusters remains to be clarified.

Recent experiments by Pierce and colleagues have provided considerable insight into the earliest events of B-cell antigen recognition and signaling $[13,49,55-58]$. For example, antigen ligation-induced stable FRET observed between BCR ectodomains (Fab fragments) indicates that BCRs are held in close proximity within a molecular cluster. The FRET between BCR cytoplasmic domains, in contrast, increased upon antigen ligation but eventually decreased resulting in a nonmonotonic behavior. It was hypothesized that antigenclustered BCRs undergo a conformational change ("closed" to "open" conformation) in their cytoplasmic domains that coincides with the initiation of phosphorylation of BCR signaling chains by Src family kinases [55]. Very recent experiments in the Pierce lab have shown that the conformational changes in the BCR cytoplasmic domains, as well as the growth of BCR microclusters, are affinity dependent [13].

As mentioned in the previous section, the theory of kinetic proofreading was invoked to explain specificity in $\mathrm{T}$ cell activation. In this model, for antigens with high dissociation constant $k_{\text {off }}$, premature dissociation of the TCR from the pMHC molecules would result in lack of T-cell activation [31]. Thus, mature $\mathrm{T}$ cells that are trained to bind selfligands with very low affinity $\left(\sim 100 \mu \mathrm{m} K_{\mathrm{D}}\right.$ or less) cannot get activated. The ligands that bind TCRs below the threshold of activation (resulting in partial/suppressed activation) can be called antagonists. In contrast, agonist ligands are able to bind to TCRs for sufficiently long time to complete the kinetic proofreading requirement leading to productive $\mathrm{T}$ cell signaling. It was shown that efficient T-cell activation takes place for an optimal dwell-time range of TCR-pMHC interaction, whereas mutations (in TCR or MHC molecules) that either decrease or increase the binding half-life (away from the optimal value) result in impaired T-cell activation [10]. Loss of serial triggering beyond an optimal half-life of TCR-pMHC interaction was implicated in such a nonmonotonic affinity discrimination. For high antigen concentration, however, their data indicates that the threshold half-life could shift towards a higher value of affinity. A recent study of Tcell activation that utilized varying affinity for antigens (using altered peptide ligands and engineered TCRs) also indicates the role of antigen concentration in T-cell affinity discrimination. For high antigenic concentration, high-affinity TCRpMHC binding ( $\sim 10 \mathrm{~nm} K_{D}$ with large TCR-pMHC halflife) seems to compete with lower affinity agonist binding [59]. The loss in serial triggering, with increasing affinity, presumably becomes less significant when a large number of antigens are present. It is possible that, in vivo, B cells present increased amount of antigen in some cases of high-affinity TCR-pMHC binding. Therefore, even if the intrinsic affinity discrimination is found to be nonmonotonic in $\mathrm{T}$ cells, it may emerge to be a strategy to compensate for increased presentation of ligands (in vivo) with increased antigenic affinity. The nonmonotonic affinity discrimination has been questioned in some recent studies of affinity discrimination in T cells $[39,40]$. In this context, it will also be interesting to explore the nature of affinity discrimination for the earliest events of T-cell activation (such as the formation of TCR microclusters; previous studies mostly focus on downstream activation events such as IL-2 secretion). Recent studies in $\mathrm{B}$ cells provided evidence of increased BCR oligomerization and microcluster formation with increased affinity [13].

Even though recent imaging experiments added to the previous biochemical and genetic data and have begun illuminating the earliest events of T- and B-cell activation, a clear and precise molecular level understanding of the initial events of lymphocyte activation and affinity discrimination is still lacking. Choosing the correct model of activation, from a set of plausible models that are proposed based on experimental data, remains challenging. In this context, several additional biological questions need to be addressed. For example, the reason why B cells can distinguish between specific and nonspecific ligands (or self and nonself) differing by only a tenfold difference in antigen affinity, whereas the same tenfold difference in antigen affinity in the high affinity range results in only slight or no increase in B-cell signaling, needs to be addressed. Recent advent of high-resolution imaging techniques provides unique opportunities for developing insightful theoretical models. Mathematical and computational models, such as the Monte-Carlo-based approaches, are well suited to (i) analyze a vast amount of experimental data and provide mechanistic insight into existing experimental observations, (ii) predict new phenomena that can be verified in further experiments, and (iii) choose the correct model of activation.

\section{Lipid Raft Formation as a Mechanism for Affinity Discrimination in Immune Cells}

Formation of stable receptor-lipid rafts, upon antigen binding, might hold the key to early time affinity discrimination in immune cells possessing multichain immune recognition receptors (MIRRs). Clearly, receptor-lipid raft formation provides a mechanism for association between immune receptors equipped with signaling chains (ITAMs) and signaling kinases such as Lyn/Lck (assuming those kinases are preferentially located in sphingolipid membrane domains). In addition, initial signaling through rafts might orchestrate the subsequent formation of immunological synapses, as observed in B and T lymphocytes. Signaling through rafts and immunological synapses, integrated over time, should determine the eventual cellular response and functional outcome of activation. Research carried out in my lab in the last few years elucidated the mechanisms of early-time affinity discrimination in B lymphocytes [15, 16, 60-65]. In our most recent effort, we developed and studied a fairly detailed Monte Carlo model of receptor-lipid raft formation in B cells. Initial results indicate affinity-dependent formation of BCRlipid rafts; signaling through BCR-lipid rafts was also found to 
be affinity dependent. Insights gained from our studies might be applicable to other types of immune cells exhibiting affinity discrimination, such as in T lymphocytes.

Before we discuss Monte Carlo simulations of BCR-lipid raft formation, previous studies of immunological rafts are briefly reviewed. Lipid rafts have been implicated as a platform for signaling in a variety of immunological problems, and its formation has been studied [66-70]. In addition, it has been implicated as a portal for viral entry into host cells and in viral manipulation of the host signaling pathways [71]. In B cells, early studies indicated rafts as the site for signaling and antigen internalization [72].

Experimental Studies of Immunological Lipid Rafts. In some of the earlier studies on B-cell lipid rafts, based on biochemical measurements, BCRs were shown to be localized in the sphingolipid rich regions of membrane upon antigen crosslinking [72-75]. In addition to BCR molecules, critical signaling molecules such as PLC $\gamma 2$ were shown to be recruited to the detergent insoluble membrane microdomains, confirming the role of lipid rafts as a signaling platform [73]. Early studies in $\mathrm{T}$ cells indicated antigen stimulation-dependent recruitment of TCR $\zeta$ and other crucial membrane proximal signaling molecules to lipid rafts [76]. In a similar manner, FceRI was shown to associate with lipid rafts in activated mast cells and basophils $[77,78]$. Doubly acetylated Src family kinases (such as Lyn and Lck) are known to constitutively present in plasma membrane inner leaflet rafts in resting immune cells. Collectively, these early studies seem to indicate that the immune receptors (BCR, TCR, and FceRI) and some of the key membrane proximal signaling molecules are recruited to lipid rafts upon stimulation, and lipid rafts act as a signaling platform $[67,72,74,75,79]$.

Dynamics of BCR-lipid raft formation was demonstrated by FRET between BCR Ig-alpha and Lyn FL/Lyn 16 (lipid raft probe) $[56,57,80]$. In addition, FRET between BCR cytoplasmic domains changed from a nonmonotonic behavior to a simple increase when either lipid rafts were disrupted by cyclodextrin or the ITAM tyrosine residues were replaced by phenylalanine [55], indicating the role of lipid rafts and BCRLyn association. DeFranco and colleagues studied lipid rafts in B cells utilizing imaging and proteomic techniques $[81,82]$. Proteomic measurements provided quantitative information regarding lipid raft localization of key signaling molecules (including BCRs) before and after antigen binding.

Study of lipid rafts in $\mathrm{T}$ cells has been a topic of intense interest and debate. In resting $\mathrm{T}$ cells (without antigenic stimulation), TCRs are located outside lipid rafts and may form oligomers [83, 84]. Coreceptors CD4 and CD8 are, however, known to be localized in resting cell rafts [85]. Antigenic ligation leads to formation of microclusters of TCRs and other signaling molecules. Previous biochemical measurements indicated that TCR signalosome is assembled in lipid rafts upon antigenic stimulation [79]. Recent superresolution studies indicate that such microclusters are formed through association of preformed TCR clusters and lipid rafts [86]. Lipid rafts have also been implicated in formation of the immunological synapse (at the cell-cell contact between a $\mathrm{T}$ cell and an antigen presenting cell). Recent studies in T-cell rafts are reviewed in $[83,86]$.

We summarize some of the experimental techniques used in studies of lipid rafts: detergent insoluble membrane fraction [72, 73], fluorescence microscopy [81], fluorescence resonance energy transfer (FRET) [56, 80, 87-89], fluorescence recovery after photobleaching (FRAP) [90], singleparticle tracking (SPT) [91, 92], two-photon microscopy [93], and super resolution techniques [94] (some references are specific for studies in immune cells). In addition, atomic force microscopy (AFM), Raman spectroscopy, birefringence microscopy, and nuclear magnetic-resonance- (NMR-) based methods can be utilized to study lipid rafts in immune cells.

Theoretical Studies of Immunological Lipid Rafts. There have been some mathematical/computational attempts to model raft formation in immune cells. Size of lipid rafts, both in resting cells and in stimulated cells (such as due to ligand binding), is a problem of considerable interest. Based on recent experimental studies, it seems likely that resting cell rafts are highly dynamic and raft sizes are small $(\sim 10 \mathrm{~nm})$ $[95,96]$; however, the size of resting cell rafts remains controversial, and a range of values for resting state raft sizes has been reported $[83,96]$. It also seems reasonable that resting state raft size could vary in a cell-type-specific manner. A Poisson-Boltzmann-based theoretical approach was used to explain $\sim 100 \mathrm{~nm}$ raft size on resting living cell membranes [97]. In addition to intrinsic tendency of raft components to segregate, a long-range dipolar interaction among membrane constituents was postulated. It is likely that intrinsic attractive interactions among similar lipids (that can drive segregation), line tension, and short-range attractive interaction among membrane constituents will be sufficient to explain small dynamic rafts (in resting cells).

An agent-based Monte Carlo simulation was utilized to study the earliest events of B-cell surface dynamics upon antigen cross-linking (by soluble multivalent antigens) [98]. Both BCR-ligand interaction and BCR-raft interaction were modeled. A hybrid simulation scheme was developed where receptor (BCR) entry to/exit from rafts was modeled using a set of ODEs; reaction rates (for entering/leaving rafts) were, however, assumed to be probabilistic. In addition, lipid raft diffusion and merging were simulated. Receptor diffusion was studied utilizing mean square displacement (MSD), which was shown to be affected by internal viscosity of the rafts (slower diffusion of molecules inside rafts) as well as by presence of raft obstacles (for molecules outside rafts). Short-term diffusion was found to be anomalous while regular diffusion was observed on a longer time scale. In this study, receptors were shown to aggregate inside rafts due to increased viscosity (slower receptor diffusion) and led to enhanced BCR-antigen complex formation in the raft domains. A key limitation in the model is that the probability for BCRs entering/leaving rafts did not depend on the state of antigen binding and preformed BCR rafts existed prior to BCR-antigen binding. Later study by the same group utilized an improved in silico model of early time $(\sim 1 \mathrm{~min})$ B-cell activation [99]. In this new model, BCR aggregation was assumed to be a key to dynamic partitioning of BCRs into 
lipid rafts regions. As BCR cross-linking by soluble multivalent antigens leads to BCR aggregation, ligand binding effectively induces increased recruitment of BCRs into lipid raft regions; BCR ligation state, however, did not directly affect raft partitioning of BCRs. Such a selective partitioning of aggregated BCRs in lipid rafts resulted in an affinitydependent increase in the following membrane proximal signaling events: (i) percentage of cross-linked BCRs and (ii) ITAM/Lyn tyrosine phosphorylation (as the avidity of antigen is increased). Affinity-dependent membrane events, as observed in this study, are consistent with other Monte Carlo studies of BCR clustering by soluble antigens [62, 63]. A virtual FRET measure was utilized to analyze simulation data and compare it with existing experimental results. For example, early-time dynamics of BCR-BCR FRET efficiency obtained from simulations correlated well with experimental FRET between BCR Fab fragments (upon stimulation with NP-BSA ligands [55]). It is possible to study affinity variation in their FRET measure (such as between BCR-BCR) and verify the results in further experiments (affinity dependence in FRET between Fab fragments has not been studied in experiments to date). Negative selection by self-ligands was addressed using the same in silico model but assuming low number of B-cell receptors as known for the pre-B-cell population (in bone marrow). Low receptor density (in their simulation) resulted in slightly decreased ligand binding but adequate signaling that was, however, not sufficient to explain negative selection. Altered partitioning of BCRs in immature $\mathrm{B}$-cell rafts (compared with that in normal B cells) is known $[75,100]$ and needs to be incorporated in a model of negative selection.

5.1. Monte Carlo Study of Receptor-Lipid Raft Formation in B Lymphocytes. Monte Carlo simulation has been increasingly used in recent years to solve biological problems [29, 30]. It essentially solves an underlying set of master equations that govern the behavior of a biological system in probability space. Simulations are carried out based on a set of predefined probabilistic rate constants (parameters of master equations) [101]. Monte Carlo simulation can handle the following key aspects of a biological process: (i) stochastic variability (such as under low concentrations of antigen), (ii) spatial heterogeneity, and (iii) biological complexity (such as complex rules of signaling regulation). In the context of modeling biological systems, it is frequently used as an agent-based stochastic modeling approach, such as in our modeling of BCR-lipid rafts. It is now established that B cells frequently recognize membrane bound antigens on the surface of antigen presenting cells [50]. In order to simulate BCR-lipid raft formation, for membrane bound antigens, we developed a hybrid Monte Carlo simulation method that combines the following approaches: (1) a probabilistic rate constant-based (implicit free energy) kinetic MC method for simulating receptor-ligand (antigen) interactions and (2) an explicit free-energy-based MC algorithm that handles BCRBCR and lipid-mediated interactions $[30,102]$. Each run of our MC simulation corresponds to a single B cell.

At the beginning of the simulation, all the molecular species are distributed uniformly and randomly on their respective 2-dimensional lattice grids. B-cell receptors need to be modeled as a two-layer structure, the bivalent Fab domains move in the top layer where it can bind monovalent antigens, and the transmembrane part diffuses in the bottom layer with lipids. Src family kinase Lyn is simulated in a manner that it can interact with lipids (Figure 2A in [30]). Lipids are placed on a smaller size lattice grid than that used for membrane proteins, but they are sampled more frequently than protein molecules to allow faster diffusion. It is possible to vary the initial lipid compositions and lipid-protein associations (in our simulations) to capture the effect of altered state of rafts observed during various developmental stages of lymphocytes [100] and in lymphocytes obtained from patients of autoimmune disorders [103, 104]. Known diffusion constants for proteins and lipids (on the plasma membrane) are used to determine the physical time scale corresponding to one MC step. This time scale is then utilized to determine the values of simulation parameters from experimentally measured kinetic rate constants (e.g., probabilistic reaction constants $P_{\text {on }}$ and $P_{\text {off }}$ are obtained from the association and dissociation rate constants $k_{\text {on }}$ and $k_{\text {off }}$ defined in (1)) $[29,30]$. It is technically challenging to simulate two disparate size objects (lipids and BCRs) placed on different size grids on the same 2-dimensional lattice.

The following free-energy-based parameters govern the diffusion of protein and lipid molecules: $\mathrm{BB}$ (between two BCRs), BL (between a BCR and a lipid), LL (between two raft forming lipids), and KL (between a Lyn and a lipid). A lattice gas-type Hamiltonian (energy function) was used to estimate the free energy in each configuration [101]. In resting cells, $\mathrm{BB}$ might arise due to interaction between the two identical sides of transmembrane alpha helical part of two receptors $[105,106]$. The value of $\mathrm{BB}$ is assumed to be small $\left(<K_{B} T\right)$ to prevent large-scale BCR clustering in resting cells. It is known from FRET studies that only a small proportion of BCRs $(\sim 20 \%)$ exists in an oligomeric state in resting B cells $[49,55]$. The value of $\mathrm{BL}$ is also assumed to be small in resting cells, as BCRs are not known to reside in sphingolipid rich domains. Upon antigen binding, however, both $\mathrm{BB}$ and $\mathrm{BL}$ are assumed to be significantly larger from their resting cell values. For membrane-antigen bound BCRs, the origin of large $\mathrm{BB}$ presumably lies in opening of the $c \mu 4$ domain of BCRs [58]. For soluble multivalent antigens, cross-linking of bivalent BCRs can be a mechanism for generating an effective large $\mathrm{BB}[62$, 63]. As for increased BL upon antigen binding, in addition to energetics and entropy (such as van der Waals interaction or hydrophobic mismatch), membrane curvature and rigidity can also be important. This will be discussed further when we describe inclusion of a time-dependent equation (PDE) for membrane shape fluctuations in our hybrid Monte Carlo model. Variations in $\mathrm{BB}$ and $\mathrm{BL}$ values, which depend on the state of antigen binding, create a coupling between the implicit free-energy and explicit free-energy components of the hybrid Monte Carlo approach. In resting cells, Src family kinase Lyn is already localized in sphingolipid rich regions (lower leaflet of the plasma membrane) due to its dual acyl chains, the effect of which could be captured by the parameter KL. Lipid-lipid interactions (LL) originate from energetics and entropy; interactions of hydrophobic origin might be 
key to generating preferential attractive interaction among raft forming sphingolipids. In our Monte Carlo model, only one type of lipid molecules (raft forming sphingolipids) is considered using a mathematical mapping. The parameter LL is an effective parameter that captures the combined effect of $L_{\mathrm{r}}+\mathrm{LL}_{\mathrm{nr}}$, where $\mathrm{LL}_{\mathrm{r}}\left(\mathrm{LL}_{\mathrm{nr}}\right)$ are attractive interactions between two raft forming (nonraft forming) lipids. Additional experimental studies, as well as molecular simulations (MD and Monte Carlo), are needed to obtain more precise values of some of the energy-based parameters and to elucidate the mechanisms of underlying physical interactions.

5.2. Simulations Results: Affinity-Dependent Formation of BCR-Lipid Rafts. After all the molecules are randomly placed on their respective lattice grids (at $t=0$ ), we allow lipidmediated interactions to generate resting cell rafts. LL values, such as that for sphingomyelin, are approximately known from previous biophysical studies [107, 108]. Similar or slightly higher (to capture the effect of cholesterol) LL values in our simulations did not generate large-scale sphingolipidenriched membrane domains (at $T=37^{\circ} \mathrm{C}$ ). Mobile protein obstacles, such as BCRs, favor entropic forces that prohibit large-scale organization of similar lipids. We observed formation of small $(<50 \mathrm{~nm})$ transient rafts consistent with what is known for resting $B$ cells. Antigen binding leads to enhanced $\mathrm{BB}$ and $\mathrm{BL}$, generation of BCR-sphingolipid rafts, and thus colocalization of BCRs with signaling kinase Lyn. BCR-Lyn association is a key signaling step as any types of activatory signal can propagate downstream only when BCR signaling chains (ITAMs) are phosphorylated by Lyn. We could carry out controlled Monte Carlo experiments (in silico) of affinity discrimination, by varying the parameter of BCR-antigen affinity $\left(K_{A}\right)$ and keeping all other parameters unaltered.

Formation of BCR-BCR oligomeric clusters was the earliest event subsequent to antigen binding [16]. Clustering of BCRs gradually transforms into BCR-lipid (raft forming sphingolipid) domains. Formation of BCR-lipid rafts was faster as the affinity for antigens increased. Average size of the formed microdomains was also bigger for higher affinity binding (Figure 2B in [30]). Transient uncoupling of actin cytoskeleton, such as due to observed dephosphorylation of ERM proteins [82], would allow formation of large receptorlipid rafts. The number of BCRs clustered in sphingolipid rich domains, as measured by total BCR-BCR adjacent pairs and BCR-lipid adjacent pairs in our simulations, monotonically increased with increasing affinity (across physiological range of five orders of magnitude) and thus provides a mechanism for affinity discrimination $[30,102]$. Thus, the time-to-raft formation and the size of receptor-lipid rafts emerge to be two key parameters that regulate affinity discrimination. For very high BB (receptor-receptor interaction energies), the size of lipid rafts is expected to decrease (beyond an optimal value for the interaction energy) [62]. Whether such a decrease in raft size is observed in B/T lymphocytes and how that would impact the affinity discrimination remains to be explored. The ratio BB to BL is also likely to be an important parameter for regulating the dynamics of raft formation and may vary depending on the cell type (such as between $\mathrm{B}$ and $\mathrm{T}$ cells).
The underlying physics of raft formation is similar to the phase separation processes in two liquid systems. BCRs and raft forming lipids can be treated as one type of liquid molecules that phase separates from other lipids and proteins. Here, antigen binding drives the system out of its steady state (small unstable rafts), similar to a low temperature quench that drives a homogeneously mixed two-liquid system out of equilibrium. As long as we do not consider any active processes, the problem of BCR-lipid raft formation is essentially an out-of-equilibrium dynamics towards an effective equilibrium state. BCRs are assumed to have significantly increased mutual attractive interactions (BB) upon antigen binding (due to opening of the $c \mu 4$ domain), leading to higher value of $\mathrm{BB}$ (on average) for higher affinity as antigens stay bound longer for such higher affinity binding. Such effective affinity dependence in $\mathrm{BB}$ (and presumably in $\mathrm{BL}$ ) introduces affinity into the problem of raft formation. In a two-liquid system, temperature determines the degree of phase separation upon a low-temperature quench. Here, affinity determines how far out of equilibrium the system is driven by antigen binding. As expected, the resulting phase diagram is also affinity dependent elucidating the physical basis of early-time affinity discrimination in B cells. Below a threshold affinity (such as for self-ligands), the phase separation in raft domains would not occur, even if the antigen concentration was taken to be very high.

\subsection{Kinetic Proofreading and Serial Triggering in a Lipid} Raft Model. We already discussed kinetic proofreading and serial triggering, concepts that have been invoked to elucidate the mechanisms of affinity discrimination in immune cells. Such concepts need to be reanalyzed in the light of in silico studies of BCR-lipid raft formation and molecular level events observed in recent experiments (during early-time activation in immune cells). Clustering of receptors (and slower diffusion) in lipid rafts can affect the way single antigens serially trigger immune cell receptors. In some of the earlier mathematical and computational studies, kinetic proofreading had to be incorporated ad hoc to explain affinity discrimination [11, 31, 109] (even though biochemical events such as ITAM tyrosine phosphorylation were mentioned as potential kinetic proofreading steps). In a model of BCR-lipid raft formation, however, kinetic proofreading can emerge naturally from the course of molecular events without any $a$ priori assumptions. In our simulations of resting B cells, BCRs are predominantly outside rafts while Lyn mostly resides in sphingolipid rich regions. Thus, BCR and Lyn are segregated into distinct spatial regions in resting $\mathrm{B}$ cells prohibiting spontaneous activation. Antigen binding, however, leads to generation of BCR-sphingolipid rafts and colocalization of BCRs with signaling kinase Lyn. The delay in BCR-Lyn association, upon antigen binding, can provide a key step in kinetic proofreading mechanism. The following antigen stimulation-induced membrane proximal events can serve as kinetic proofreading steps: (1) dimer/oligomer formation of BCRs, (2) BCR-lipid raft formation and BCR-Lyn colocalization in stable rafts, (3) phosphorylation of BCR ITAM tyrosine residues by Lyn (presumably leading to "closed" to "open" transition in BCR cytoplasmic domains [55]), and (4) 
recruitment of Syk to BCR cytoplasmic domains. In a similar manner, in resting $\mathrm{T}$ cells (without antigen stimulation), TCRs are located outside lipid rafts. Doubly acetylated Src family kinase Lck (equivalent of Lyn in B cells) is known to be constitutively present in the inner leaflet rafts in resting T cells. Coreceptors CD4 and CD8 are also known to be localized in resting cell rafts [85]. Thus, selective partitioning and recruitment of signaling molecules, in different spatial regions of the $\mathrm{B} / \mathrm{T}$ cell membrane, can emerge as a basis for kinetic proofreading. Surprisingly, in B cells, the requirement of kinetic proofreading time decreases with increasing affinity thus favoring increasingly higher affinity for antigens.

The precise dependence of kinetic proofreading on antigenic affinity, however, is expected to vary depending on the details of the system (such as cell type) under consideration. It will be interesting to explore how differences in molecular level events can differentially coordinate receptor-lipid raft formation in different immune cells (such as B and T lymphocytes) leading to distinct patterns of affinity discrimination. Some of the energy-based parameters (such as BB and BL), for example, can vary depending on the immune cell type under consideration. In $\mathrm{T}$ cells, recent studies indicate the presence of significant amount of TCR clustering in resting naïve $\mathrm{T}$ cells [83] (though contradictory results exist [110, 111]). If such TCR clusters (protein islands/nanoclusters) exist in resting $\mathrm{T}$ cells that would affect the kinetic proofreading step of TCR-Lck colocalization. It will also be interesting to explore if the BCR clustering differs from the TCR clustering (in resting cells) and whether such differences contribute to differences in affinity discrimination behavior between $B$ and T cells. Other possible mechanisms such as more severe loss of serial triggering in $\mathrm{T}$ cells (with increasing affinity), than that in B cells, could also contribute to nonmonotonic discrimination observed in T cells. Clearly, the pMHC-mediated antigen binding mechanism in $\mathrm{T}$ cells differs from BCRs binding to whole antigens. Even at the level of the molecular organization, the TCR CD3 complex differs significantly from the BCR $\operatorname{Ig} \alpha-\operatorname{Ig} \beta$, which may lead to differences in the formation mechanism of dimeric/oligomeric clusters. In addition, coreceptor signaling, Src family kinase recruitment, coagonism of self-peptides (endogenous peptides), and receptor downregulation can be important in TCR serial triggering and affinity discrimination; all these factors, however, should impact the early-time T-cell affinity discrimination through the formation of TCR-lipid rafts. Early-time feedback regulations (such as through ERK and SHP-1) can also be important in generating affinity discrimination [112].

It is interesting to note that self- (endogenous) peptides were shown to act as coagonists in $\mathrm{T}$ cells (in the presence of agonist foreign ligands), and the effect was enhanced for stronger agonists [36-38]. It was also observed that the density of self peptide-MHC ligands can be a key regulator of TCR signaling [38]. Therefore, the mode of ligand presentation, such as clustering of pMHC or antigen on the antigen presenting cells, can impact receptor-induced signaling and affinity discrimination. Study of different modes of ligand presentation can also be significant in the context of vaccine design as novel nanotechnology-based approaches [113] will provide various options for controlled presentation of antigens.

5.4. Coupling Membrane Mechanics with Receptor-Ligand Binding and Lipid-Mediated Interactions: Implications for Receptor-Lipid Raft Formation. The change in the local intermembrane membrane separation distance $z$ is assumed to evolve (towards the free-energy minimum) according to a time-dependent Landau-Ginzburg formulation [114-118]:

$$
\frac{\partial z}{\partial t}=M\left(-\sum_{i=1}^{N} \kappa_{i} C_{i}\left(z-z_{e q(i)}\right)+\gamma \nabla^{2} z-\beta \nabla^{4} z\right)+\zeta
$$

The local membrane shape is described by $z(x, y)$. For two cells in contact, $z$ denotes the intermembrane separation distance. The first integral in the equation contains the energy associated with stretching of receptor-ligand bonds, which is a function of the local concentration $C_{i}(x, y)$ of $N$ species of receptor-ligand bonds (such as BCR-antigen complexes), where $\kappa_{i}$ and $z_{\text {eq }(i)}$ are the bond stiffness $(\mu \mathrm{N} / \mathrm{m})$ and equilibrium bond length of species $i$, respectively. The last two terms relate to the energy associated with membrane tension $(\gamma)$ and bending rigidity $(\beta)$, respectively. Previously we could incorporate the effect of membrane shape fluctuations into our kinetic Monte Carlo model for receptor-ligand binding [64]. Uniform values for the membrane parameters $(\gamma, \beta)$ were assumed over the entire membrane. However, one may assign two distinct sets of membrane parameters, $\left(\gamma_{1}, \beta_{1}\right)$ and $\left(\gamma_{2}, \beta_{2}\right)$, corresponding to sphingolipid and phospholipid rich regions, respectively. It will be interesting to explore if receptor-ligand binding and BCR-BCR oligomerization (due to $c \mu 4$ opening) can lead to preferential localization of BCRs into stiffer sphingolipid rich regions (presumably having higher $\gamma$ and $\beta$ ). In this manner, the BL parameter mentioned earlier in this paper (Section 5.1) can partially emerge from a coupling between membrane mechanics and receptorligand binding. There might be other interesting effects that would result from linking membrane mechanics with receptor-ligand binding and lipid-mediated interactions. Transient uncoupling of actin cytoskeleton from BCR-lipid raft domains [82] can lead to increased membrane flexibility (decreased bending rigidity). In addition, opening of the $c \mu 4$ domain might acquire some sort of spatial dependence if the pulling force (that causes $c \mu 4$ opening) on BCRs depends on the intermembrane separation.

5.5. Functional Role of Rafts: Affinity Discrimination in Immune Cells. Functional significance of lipid rafts, such as those exist on the resting cell plasma membrane or activation induced, remains a key question in the field of raft research. It is becoming increasingly clear that rafts can allow signaling molecules to assemble in a signaling platform (signalosome) for initiation and modulation of specific cellular signaling. In some other situations, disruption of rafts might actually lead to enhanced signaling. For example, some studies indicate that growth receptor EGFRs can form dimers more easily once those are outside rafts [119]. Formation of EGFR dimers, 
upon EGF binding, is known to be a key step in the initiation of autophosphorylation and signaling. In B cells, some earlier studies found that disruption of rafts by cholesterol depleting agents (methyl- $\beta$ cyclodextrin) can enhance signaling through BCR signaling chains. Whether such cholesterol depletion-mediated enhancement of signaling is robust across physiological range of affinity values and under variation in BCR/antigen density remains to be seen. What is then the biological function of B-cell immune rafts? Clearly, it provides a mechanism for protecting resting $\mathrm{B}$ cells from spontaneous activation by segregating BCRs and Lyn in distinct membrane locations. It is also evident that rafts can be considered as signaling regulators that can selectively recruit/remove signaling (as well as inhibitor) molecules, in a dynamic manner. For low concentration of antigens, or for low-affinity binding, spatial clustering in raft domains may boost an otherwise weak signaling (by satisfying kinetic proofreading requirement and increasing serial triggering). For antigen-induced strong signaling, on the other hand, rafts might function to downregulate the signaling. Therefore, it is expected that lipid rafts in B cells function to adjust the membrane proximal signaling response to the antigen load and affinity. It is also possible that rafts orchestrate the signaling in a dynamic manner: an initial weak signaling is enhanced but downmodulated once a threshold is reached (a similar mechanism has been proposed for signaling through the T-cell immunological synapse [120]). For small number of antigens/BCRs, or for low antigen affinity, spatial clustering in rafts can also be a mechanism for reducing cell-to-cell stochastic variability.

Our initial findings, interestingly, indicate that BCR-Lyn association is regulated by affinity-dependent formation of large stable receptor-lipid rafts (upon antigen ligation) providing a mechanism for kinetic proofreading and affinity discrimination. Clustering of BCRs in raft domains should also regulate serial triggering by antigens, especially when the antigen affinity is low and rapid dissociation of receptorantigen bonds is possible (such as during the early phase of an infection). Thus, the functional role of BCR-lipid rafts emerges to be more intricate than simply providing a platform for signaling. It is now increasingly recognized that the lipid and/or cholesterol composition in a cell can be altered to regulate signaling outcome. In some types of B cells (such as autoimmune or memory B cells), altered lipid composition and lipid-protein association may lead to faster and stronger response to antigenic stimulation. It seems reasonable to expect that receptor-lipid rafts would function in a similar manner to achieve affinity discrimination in other immune cells equipped with MIRRs (multichain-immunorecognition receptors), such as in T cells. There might be other examples in cell biology where important cellular function is intricately linked to the formation mechanism of rafts imparting highly specialized functional role to lipid rafts. Given the key importance of receptor-lipid rafts in immune cell activation, especially in the affinity discrimination process, study of immune rafts is likely to be an area of intense future research. Advent of novel experimental technologies, such as the superresolution method, should bring new excitements to this emerging area of study.

\section{Mathematical and Computational Modeling of Raft Formation in Adaptive Immune Cells: A Tool to Analyze Recent Experimental Results}

Monte-Carlo-based in silico study of BCR-lipid raft formation can be utilized to analyze recent data gathered by experimental studies of B-cell activation. For B lymphocytes, data is available from imaging experiments, FRET studies, and proteomic studies $[56,80,82]$. It is possible to integrate this diverse set of experiments through mathematical and computational studies. One can define a set of different quantitative measures for analyzing simulation results. Each of those quantitative measures then can be utilized to analyze a specific type of experimental data. For example, a measure for BCR-Lyn association can be useful in analyzing dynamical FRET between Lyn FL/Lyn16 (lipid raft probe) and BCR cytoplasmic domains [80]. Estimation of the BCR fraction localized in sphingolipid domains before and after antigen ligation, from BCR-lipid raft simulations, can be compared with data obtained from proteomic measurements in [82] (or similar measurements). Another study of lipid rafts in B lymphocytes, based on biochemical measurements, indicated that disruption of rafts (by methyl-beta-cyclodextrin) can actually lead to increased signaling. In the Monte Carlo model, one can potentially study the effect of raft disruption by changing some of the parameter values (such as by decreasing the lipid-lipid interaction parameter LL described in Section 5.1). In the experimental studies of receptor-lipid raft formation in B cells, the effect of antigen affinity variation is not explored to date. However, such affinity variation studies could be key to elucidating the functional role of lipid rafts in B lymphocytes. A recent study explored the mechanism of early-time B cell affinity discrimination at a fundamental molecular level [13], though it did not probe the formation of BCR-lipid rafts. Two key observations that emerged from this study: (i) formation of BCR microclusters (upon antigen binding) is affinity-dependent; (ii) FRET efficiency between BCR cytoplasmic domains shows a nonmonotonic (temporal) behavior in an affinity-dependent manner. Further probing of BCR microclusters might reveal colocalization of raft forming sphingolipids in those microclusters. The nonmonotonic behavior in FRET was thought to indicate a conformational change (from "closed" to "open" form) in the BCR cytoplasmic domains that coincided with recruitment of signaling kinase Syk to the BCRs. The decay curve for the FRET between BCR cytoplasmic domains (after the FRET peak is reached), when normalized by the peak FRET, seems to indicate a simple exponential behavior; such an exponential decay can thus be analyzed by a first-order decay kinetics: $d E / d t=-k E$, where $E$ denotes the normalized FRET efficiency. Interestingly, the decay constant seems to depend on the antigen affinity indicating that $k$ is presumably determined by the degree of BCR-Lyn association. Monte Carlo simulations can be carried out to provide insight into such FRET data; initial results indicate that (Section 5.2) BCR-BCR clustering and BCR-Lyn association (through formation of BCR-lipid rafts) are both affinity dependent. 


\section{Role of Rafts in Long-Time Affinity Discrimination: Affinity-Dependent Formation and Function of Immunological Synapses}

Biochemical engineering of membrane organization and heterogeneity (based on underlying physics of lipid and protein interactions) in adaptive immune cells and the biological function of affinity discrimination are intricately linked. Clustering of receptors and signaling molecules in lipid rafts and immune synapses are two prime examples of such membrane organization and heterogeneity. Lipid rafts and immunological synapses should coordinate serial triggering and kinetic proofreading in a cell-type-specific manner and induce affinity discrimination.

After its initial discovery in T cells $[121,122]$, exploration of formation mechanism and functional role of immunological synapses has attracted a lot of attention. In T cells, it has been observed that two types of receptor-ligand pairs (TCRpMHC and LFA1-ICAM1) cluster at the cell-cell contact (between a T cell and an APC) but are also known to segregate in the form of an immunological synapse. It was later found that the synaptic pattern in $\mathrm{T}$ cells was driven by receptorligand pair length differences [114-116, 118]. Cytoskeletaldriven active transport (of receptors), though not essential, was also thought to be important in rapid ( $<5$ minutes) generation of T cell synapses $[115,117]$. In B cells, similar canonical synapse pattern (between BCR-antigen and LFA1-ICAM1) was discovered $[123,124]$ but the formation mechanism was unclear. Monte-Carlo-based studies elucidated that directed transport of $\mathrm{B}$-cell receptors (or receptor-ligand bonds) is a potential formation mechanism for B-cell synapses [60, $61,64]$, as there is no indication that there exists significant difference in equilibrium bond lengths between BCR-antigen and LFA1-ICAM1 (at least for IgM BCRs) that are forming the synaptic pattern. Thus, it is reasonable to expect that a combination of (i) difference in equilibrium bond lengths (and/or other bond properties) of two types of receptorligand pairs and (ii) cytoskeletal-driven active transport mechanisms coordinates immunological synapse formation in B and T cells. Relative significance of those two factors, in synapse formation, should depend on the cell type (also the receptor type) under consideration.

Even though some of our initial studies indicated the significance of antigen affinity in B-cell synapse formation, the role of affinity in synapse formation remains little explored to date. For example, cytoskeletal-driven directed transport of BCRs could potentially be affinity-dependent leading to affinity dependent generation of immunological synapses. It is clear from experimental studies that formation of receptor-lipid rafts precedes the formation of immunological synapses (in lymphocytes). If signaling through receptorlipid rafts is affinity dependent, as emerges from our recent simulations of raft formation in B cells, it could generate affinity-dependent directed transport. Besides regulating the directed transport of receptors, early-time receptor-lipid rafts might be impacting the formation of immunological synapse in various other interesting ways. Immunological synapse might itself be enriched in sphingolipid and cholesterol. In $\mathrm{T}$ cells, participation of rafts in immunological synapse formation remains controversial based on initial studies [79]. Further studies are needed to link receptor-lipid rafts and immunological synapses. This seems to be an exciting future area of research that can benefit both raft research as well as the field of immunological synapses.

Whether or not rafts are integral part of an immunological synapse, long-time signaling and affinity discrimination should depend on function of immunological synapses, as those synaptic structures are known to be stable over an hour (or even longer). In $\mathrm{T}$ cells, based on an combined experimental and theoretical study, it was proposed that immune synapse boosts initial signaling but also leads to severe loss in signaling (due to increased TCR degradation) at long times; impairment in T-cell synapse formation in that study leads to maintenance of strong signaling over long time [120]. While the finding of signaling attenuation by immunological synapse seems to be a major function of the synapse, it seems more reasonable that early-time strong signaling is achieved through lipid rafts. Whether T-cell synapse can boost an initial signaling needs to be elaborated by further studies, but might be important for low number of antigens and/or for low-affinity binding [125]. Clustering of receptors (in the immune synapse) can definitely affect the way single antigens serially trigger immune receptors. In this context, the mechanism of receptor (BCR/TCR) downregulation and the role of self-antigens also need to be considered. In B cells, for example, internalization of BCR-antigen complex should not free up single antigens for increased serial triggering (compare $n_{a} / n_{r}$ with $\left(n_{a}-1\right) /\left(n_{r}-1\right), n_{a}$ : number of antigens and $n_{r}$ : number of receptors). Supporting the hypothesis of affinity discrimination through immunological synapses, it was shown that B-cell synapse collects antigens (in the synapse) in an affinity-dependent manner [12]. This finding has important ramifications for affinity discrimination in $\mathrm{T}$ cells (in vivo) and will be discussed further in the next section.

\section{Affinity Discrimination In Vivo}

Only a few specific lymphocytes (B and $\mathrm{T}$ cells), out of billions of such cells, are known to get activated and mount a successful immune response against a particular type of pathogen. How the adaptive immune system carry out such a task remains a problem of considerable interest. It is now established that both $\mathrm{B}$ and $\mathrm{T}$ lymphocytes first encounter antigen on antigen presenting cells (such as dendritic cells) for initial priming; afterwards B lymphocytes present antigen to $\mathrm{T}$ cells during which both of those cells receive a second activatory signal (for thymus-dependent antigens). Our initial studies on B-cell synapse formation, as well as experimental studies, indicate that B cells collect antigen in the synapse in an affinity-dependent manner. Thus, B-cell internalization of antigens and subsequent presentation to T cells presumably increase with increasing affinity. Hence, any study of affinity discrimination in $\mathrm{T}$ cells should account for increasing level of antigens presented by B cells equipped with higher affinity receptors. Affinity-dependent presentation of antigens by $\mathrm{B}$ cells might also have interesting consequences on the 
affinity maturation process, which is partly mediated by repeated interaction between $\mathrm{B}$ and $\mathrm{T}$ lymphocytes. Whether increasing levels of antigens presented to T lymphocytes, by affinity matured B cells, can compensate for decreased $\mathrm{T}$ cell signaling for high-affinity (beyond an optimal threshold) antigens remains an interesting question for further exploration. Some earlier studies indicate existence of T-cell affinity maturation by selective expansion of high-affinity $\mathrm{T}$ cell receptors [126-129] (from the existing pool of TCRs as there is no evidence of TCR somatic mutation).

\subsection{Affinity Discrimination and Affinity Maturation. B cells} have the ability to optimize the immune response against changing pathogenic load by utilizing the process of affinity maturation during which B-cell receptors with increasingly higher affinity for antigens are clonally selected. Such an optimization process can be a key to adaptability of the immune system. In general, biological systems (such as biological networks) are engineered for an optimal response, and the nature of optimization should depend on the function of the biological system under consideration. Previous theoretical studies explored optimal strategies in immunology [130132], including the optimal control mechanism for somatic hypermutation and affinity maturation $[133,134]$. It will be interesting to study the problem of affinity maturation as a process that tries to minimize antigen load (maximize antigen-antibody reaction for antigen elimination) utilizing a random process of generating BCR diversity. It seems likely that a possible solution to this problem will require some type of feedback mechanism that can regulate the mutation rate (as the pathogenic load varies). It has been proposed that antigen-antibody complex formation can interact with the complement system and thereby provides an important signal for inducing somatic mutations in germinal center B cells. The antigen-antibody-complement complex delivers this positive regulatory signal by cross-linking the antigen receptor with coreceptor (CD21/CD19/CD81) molecules. Such an antigen-antibody-complement complex-mediated signaling should be highly affinity dependent and presumably coordinated through receptor-lipid rafts. FRET-based measurements have indicated that colligation between the BCR and the coreceptor (CD19/CD21) prolongs BCR association with lipid rafts [56]. How in vivo feedback mechanisms utilize the somatic mutation rate to optimize the response against a given pathogenic load remains a challenging problem to tackle. With the recent advances in imaging techniques that are able to visualize both cellular and molecular level mechanisms of B-cell activation (such as raft clustering) as well as complex details of in vivo interactions, the field is poised for such fundamental elucidations.

Affinity maturation remained a problem of considerable interest to immunologists. Linking affinity maturation to single-cell affinity discrimination in B cells, however, is more recent. The relation between affinity discrimination and affinity maturation is intricate. It is clear that monotonic affinity discrimination observed in B cells will provide advantage to higher affinity BCRs (generated by the affinity maturation process) in generating increasingly stronger immune response, elucidating the biological function of affinity maturation. Interestingly, higher activation level of B lymphocytes (with increasing affinity) can itself be a mechanism that leads to affinity maturation. B-cell affinity discrimination can impact the selection of high-affinity receptors during affinity maturation in the following manner.

(1) B cells can receive stronger second signal of activation from $\mathrm{T}$ cells by presenting increasing amount of antigen (acquired by higher affinity BCRs). Increasing T-cell-mediated signaling has been implicated in the affinity maturation process [27]. T cells also presumably receive a stronger activation signal due to the larger amount of antigens presented. Such a process can generate a mutual feedback effect due to repeated interaction between $\mathrm{B}$ and $\mathrm{T}$ cells in the germinal center. T lymphocytes, though do not posses an inherent ability to generate high-affinity receptors (through somatic mutation), might be able to generate increasingly stronger response by B-cell-mediated effects.

(2) Advent of multiphoton imaging techniques has provided us with new information on the in vivo trafficking of lymphocytes (such as in lymph nodes) [135139]. This new data has attracted a lot of recent attention from immunologists [27, 140]; computational modeling studies that are synergistic with imaging experiments provided valuable insight into in vivo immune activation [141-144]. There have been some initial studies that addressed the process of B-cell affinity maturation in light of the recent in vivo imaging data $[136,141]$. It is expected that it will be a topic of high interest in the field due to its fundamental significance in adaptive immunity as well as for its ramifications in vaccine design. In this context, it will be interesting to explore if trafficking of B cells (such as random walk migration) can be affinity dependent and whether that affects the affinity maturation process.

(3) Apoptotic cell death has been shown to play a significant role in the affinity maturation process [27]. B cells with low-affinity receptors are shown to be negatively selected in the germinal center but can be rescued by overexpression of Bcl-2-like antiapoptotic proteins [145]. Independently, there have been recent novel findings in the system level regulatory mechanisms of apoptosis signaling [146-150]. Mathematical analysis and Monte-Carlo-based in silico studies proved to be useful in elucidating cell-to-cell stochastic variability in apoptosis signaling $[146,151]$. Such systems level information in apoptosis regulation can provide new ways to think about the role of apoptosis in affinity maturation. In addition, these findings in apoptosis signaling can impact the study of important immunological processes such as the negative selection of immature lymphocytes.

A key challenge in the field will be to develop mathematical and computational models of in vivo affinity maturation that 
can incorporate the following components: (i) competition for antigens, (ii) B- and T-cell interaction, (iii) trafficking of immune cells, (iv) apoptotic cell death, and (v) spatial heterogeneity and architecture of tissues.

\section{Affinity Discrimination and Lipid Rafts in Health and Diseases: Autoimmune Disorders and Vaccine Design}

Affinity discrimination and formation of receptor-lipid rafts in immune cells emerge to be highly connected problems. Any immunological processes (including their dysregulations), which are linked to affinity discrimination, are potentially affected by the formation of receptor-lipid rafts. Autoimmune disorders and vaccine design are two prominent examples where affinity discrimination and affinity maturation are keys to immunological activation.

Autoimmune disorders can be thought to arise from dysregulated affinity discrimination resulting in activation by lower affinity self-antigens. In SLE (systemic lupus erythematosus), for example, hyperactive immune response leads to loss in tolerance against self-antigens characterized by autoantibody production and inflammatory cells (including $\mathrm{T}$ cells) infiltration in target organ. In addition to its clear relevance in human health, study of autoimmune disorders can provide important insight into the question of self/nonself discrimination in immunology. This problem of self/nonself discrimination can be approached in two different manners: (1) explore the mechanisms by which nonself-ligands activate the immune system but self-ligands maintain tolerance; (2) how tolerance is broken in autoimmune disorders. In both of these cases, lipid rafts might be key orchestrators of immune cell activation. Recent studies indicated the crucial role of lipid rafts in SLE [103]. B cells from SLE patients showed distinct raft localization of signaling molecules (Lyn kinase and CD45 phosphatase) compared to those in healthy controls. There was significant reduction in Lyn localization in sphingolipid domains of B cells from SLE patients, whereas CD45 (low molecular weight isoform) showed enhanced recruitment to raft domains. Dynamical recruitment of Lyn and CD45 in-and-out of sphingolipid rafts, upon antigenic stimulation, showed altered kinetics. In SLE T cells, similar altered raft localization of membrane proximal signaling molecules, such as decreased raft colocalization for the kinase Lck, has been observed [104, 152]. In addition, rafts were found to be preclustered rafts in SLE T cells [104]. In B cells obtained from SLE patients, intense but irregular staining of lipid rafts (by cholera-toxin-B) was observed (compared to more uniform and light staining observed in B cells of healthy controls) [103]. As more advanced super-resolution imaging techniques become available [94], we will be able to better distinguish between small unstable rafts in normal immune cells ( $\mathrm{T}$ and B lymphocytes) and preclustered rafts in SLE lymphocytes (resting state). With our enhanced knowledge of autoimmune disorders at the cellular level, one would expect that raft-targeted therapies will be increasingly common in treatment of those diseases [153]. Mathematical and computational modeling can be important in designing optimal strategies to target lipid rafts in patients with autoimmune disorders. Monte Carlo simulations, for example, can be utilized to carry out parametric variation study (to simulate disease-specific conditions) in a rapid manner. In addition to its clear benefit in health studies, probing the altered state of rafts in lymphocytes obtained from autoimmune patients can provide unique opportunities to study the fundamental problem of affinity discrimination. Not only such studies will elucidate the mechanisms of activation against weak selfligands, but can also provide crucial information on immune activation and affinity discrimination for the entire physiological range of affinity values. One might ask, for example, whether the monotonically increasing affinity discrimination observed in B cells will still be maintained in SLE rafts (or other types of altered rafts in autoimmune disorders).

Generation of high-affinity antibodies, through affinity maturation, is a key to design of vaccines that rely on B-cellmediated antibody production and memory cell generation. Recent focus on developing vaccines that would generate broadly neutralizing antibodies against infectious agents underscores the role of affinity maturation. In the case of influenza virus, germline-encoded precursors of antibodies that bind to invariant structures on influenza haemagglutinin (HA) did not bind HA as soluble immunoglobulins (IgG), but elicited tyrosine kinase signaling (against HA) when expressed as cell surface IgM [3]. Clearly, affinity matured soluble antibodies are needed for efficient elimination of influenza HA. Thus, fundamental understanding of the initial events of B-cell signaling and affinity discrimination, as well as of the process of in vivo affinity maturation, can be critical to rational design of vaccines. A recent study to develop HIV-1 gp120 vaccine also implicated the role of affinity maturation in generating protective antibodies and suggested that improved vaccination strategies are needed to generate broadly neutralizing affinity matured antibodies [2]. Recent advent of nanotechnology-based approaches in vaccine design, such as presenting antigens on nanoparticles [113], can potentially lead to novel strategies for utilizing affinity discrimination and affinity maturation. Evidence exists that different adjuvants affect the affinity maturation process differently [154]. If mathematical and computational models can be developed to perform in silico simulations of in vivo immune activation under different immunogen/adjuvant combinations, such a tool can be utilized to optimize the efficacy of different vaccination strategies and compare among potential options. In this context, targeting the lipid rafts might open up another avenue in the field of vaccine design.

\section{Concluding Remarks}

With the advent of modern experimental technologies and recent increase in computing power, we are in a position to explore complex biological systems. The large number of degrees of freedom, involvement of multiple length and time scales, complicated structure of the interaction network among its components, and intricate rules of regulation all contribute to the complexity of a biological system. Such complexity, however, renders a biological system respond to wide variety of stimuli and adapt to a changing environment. 
Frequently, such adaptive features emerge from complex interaction among its components. In the adaptive immune system, huge diversity of antigen-specific immune receptors $\left(\sim 10^{12}\right)$, coupled with clonal expansion of high-affinity (antigen specific) receptors and memory cell generation, generates a collective fitness that dynamically evolves to impart adaptability. However, this huge diversity of immune receptors (for broad coverage of antigenic epitope space) leaves only a few specific lymphocytes for a particular antigen. Therefore, it is important to know how only a few specific lymphocytes (out of billions of such cells) can respond and eliminate foreign pathogens. Affinity discrimination and affinity maturation are two linked properties that allow the immune system to achieve this complex task; those features are also the key to adaptation. Mathematical and computational modeling has proved to be an essential tool in elucidating the mechanisms of affinity discrimination and affinity maturation. Starting from ODE-based models of kinetic proofreading to PDEbased exploration of competition between kinetic proofreading and serial triggering in affinity discrimination and to detailed Monte Carlo simulation (agent-based stochastic model that has its basis in probabilistic master equations) of immune cell activation, mathematical/computational modeling has considerably evolved over the years. It has become increasingly synergistic to experimental studies and also a necessary tool to analyze experimental data. In addition to its profound role in elucidation of fundamental biology of immune cell activation, mathematical and computational modeling might be increasingly utilized in designing immunotherapeutic strategies such as in vaccine development.
Abbreviations
BCR: B-cell Receptor
TCR: T-cell Receptor
MHC: Major histocompatibility complex
pMHC: Peptide (bound) major histocompatibility complex
ODE: Ordinary differential equation
PDE: Partial differential equation
APC: Antigen presenting cell
MIRR: Multichain-immune-recognition receptor
FRET: Fluorescence resonance energy transfer.

\section{Acknowledgment}

The author acknowledges the funding support from NIH Grant 3-CB70442.

\section{References}

[1] T. J. Kindt, B. A. Osborne, and R. A. Goldsby, Kuby Immunology, Macmillan, New York, NY, USA, 2006.

[2] M. A. Moody, N. L. Yates, J. D. Amos et al., "HIV-1 gp120 vaccine induces affinity maturation in both new and persistent antibody clonal lineages," Journal of Virology, vol. 86, no. 14, pp. 74967507, 2012.

[3] D. Lingwood, P. M. McTamney, H. M. Yassine et al., "Structural and genetic basis for development of broadly neutralizing influenza antibodies," Nature, vol. 489, no. 7417, pp. 566-570, 2012.

[4] S. Khurana, D. Frasca, B. Blomberg, and H. Golding, "AID activity in B cells strongly correlates with polyclonal antibody affinity maturation in-vivo following pandemic 2009-H1N1 vaccination in humans," PLoS Pathog, vol. 8, no. 9, Article ID e1002920, 2012.

[5] C. A. Siegrist, M. Pihlgren, C. Tougne et al., "Co-administration of $\mathrm{CpG}$ oligonucleotides enhances the late affinity maturation process of human anti-hepatitis B vaccine response," Vaccine, vol. 23, no. 5, pp. 615-622, 2004.

[6] A. M. Barkoff, K. Grondahl-Yli-Hannuksela, J. Vuononvirta, J. Mertsola, T. Kallonen, and Q. He, "Differences in avidity of IgG antibodies to pertussis toxin after acellular pertussis booster vaccination and natural infection," Vaccine, vol. 30, no. 48, pp. 6897-6902, 2012.

[7] F. D. Batista and M. S. Neuberger, "Affinity dependence of the B cell response to antigen: a threshold, a ceiling, and the importance of off-rate," Immunity, vol. 8, no. 6, pp. 751-759, 1998.

[8] V. Kouskoff, S. Famiglietti, G. Lacaud et al., "Antigens varying in affinity for the B cell receptor induce differential B lymphocyte responses," Journal of Experimental Medicine, vol. 188, no. 8, pp. 1453-1464, 1998.

[9] F. D. Batista and M. S. Neuberger, "B cells extract and present immobilized antigen: Implications for affinity discrimination," EMBO Journal, vol. 19, no. 4, pp. 513-520, 2000.

[10] A. M. Kalergis, N. Boucheron, M. A. Doucey et al., "Efficient T cell activation requires an optimal dwell-time of interaction between the TCR and the pMHC complex," Nature Immunology, vol. 2, no. 3, pp. 229-234, 2001.

[11] D. Coombs, A. M. Kalergis, S. G. Nathenson, C. Wofsy, and B. Goldstein, "Activated TCRs remain marked for internalization after dissociation from pMHC," Nature Immunology, vol. 3, no. 10, pp. 926-931, 2002.

[12] S. J. Fleire, J. P. Goldman, Y. R. Carrasco, M. Weber, D. Bray, and F. D. Batista, "B cell ligand discrimination through a spreading and contraction response," Science, vol. 312, no. 5774, pp. 738741, 2006.

[13] W. Liu, T. Meckel, P. Tolar, H. W. Sohn, and S. K. Pierce, "Antigen affinity discrimination is an intrinsic function of the B cell receptor," Journal of Experimental Medicine, vol. 207, no. 5, pp. 1095-1111, 2010.

[14] J. Ye, E. S. Bromage, and S. L. Kaattari, "The strength of B cell interaction with antigen determines the degree of IgM polymerization," Journal of Immunology, vol. 184, no. 2, pp. 844-850, 2010.

[15] P. K. Tsourkas, W. Liu, S. C. Das, S. K. Pierce, and S. Raychaudhuri, "Discrimination of membrane antigen affinity by B cells requires dominance of kinetic proofreading over serial engagement," Cellular \& Molecular Immunology, vol. 9, no. 1, pp. 62-74, 2012.

[16] P. K. Tsourkas, C. D. Somkanya, P. Yu-Yang, W. Liu, S. K. Pierce, and S. Raychaudhuri, "Formation of BCR oligomers provides a mechanism for B cell affinity discrimination," Journal of Theoretical Biology, vol. 307, pp. 174-182, 2012.

[17] G. I. Bell, "Cell-cell adhesion in the immune system," Immunology Today, vol. 4, no. 8, pp. 237-240, 1983.

[18] B. Goldstein, J. R. Faeder, and W. S. Hlavacek, "Mathematical and computational models of immune-receptor signalling," Nature Reviews Immunology, vol. 4, no. 6, pp. 445-456, 2004.

[19] T. A. Y. Shih, E. Meffre, M. Roederer, and M. C. Nussenzweig, "Role of BCR affinity in T cell-dependent antibody responses in vivo," Nature Immunology, vol. 3, no. 6, pp. 570-575, 2002. 
[20] T. A. Y. Shih, M. Roederer, and M. C. Nussenzweig, "Role of antigen receptor affinity in $\mathrm{T}$ cell-independent antibody responses in vivo," Nature Immunology, vol. 3, no. 4, pp. 399-406, 2002.

[21] J. Ye, I. M. Kaattari, and S. L. Kaattari, "The differential dynamics of antibody subpopulation expression during affinity maturation in a teleost," Fish and Shellfish Immunology, vol. 30, no. 1, pp. 372-377, 2011.

[22] P. K. A. Mongini, C. A. Blessinger, and J. P. Dalton, "Affinity requirements for induction of sequential phases of human $B$ cell activation by membrane IgM-cross-linking ligands," Journal of Immunology, vol. 146, no. 6, pp. 1791-1800, 1991.

[23] B. P. O'Connor, L. A. Vogel, W. Zhang et al., "Imprinting the fate of antigen-reactive B cells through the affinity of the B cell receptor," The Journal of Immunology, vol. 177, no. 11, pp. 77237732, 2006.

[24] M. J. Benson, L. D. Erickson, M. W. Gleeson, and R. J. Noelle, "Affinity of antigen encounter and other early B-cell signals determine B-cell fate," Current Opinion in Immunology, vol. 19, no. 3, pp. 275-280, 2007.

[25] B. M. Kessler, P. Bassanini, J. C. Cerottini, and I. F. Luescher, "Effects of epitope modification on $\mathrm{T}$ cell receptor-ligand binding and antigen recognition by seven $\mathrm{H}-2 \mathrm{~K}(\mathrm{~d})$-restricted cytotoxic $\mathrm{T}$ lymphocyte clones specific for a photoreactive peptide derivative," Journal of Experimental Medicine, vol. 185, no. 4, pp. 629-640, 1997.

[26] J. Ye, E. Bromage, I. Kaattari, and S. Kaattari, “Transduction of binding affinity by B lymphocytes: a new dimension in immunological regulation," Developmental and Comparative Immunology, vol. 35, no. 9, pp. 982-990, 2011.

[27] G. D. Victora and M. C. Nussenzweig, "Germinal centers," Annual Review of Immunology, vol. 30, pp. 429-457, 2012.

[28] D. A. Lauffenburger and J. J. Linderman, Receptors: Models for Binding, Trafficking, and Signaling, Oxford University Press, New York, NY, USA, 1996.

[29] S. Raychaudhuri, P. K. Tsourkas, and E. Willgohs, "Computational modeling of receptor ligand binding and cellular signaling processes," in Fundamental Concepts in Biophysics, T. Jue, Ed., Humana Press, New York, NY, USA, 2009.

[30] S. Raychaudhuri, "Kinetic Monte Carlo simulation in biophysics and systems biology," in Theory and Applications of Monte Carlo Simulations, W. K. V. Chan, Ed., InTech Open Access Publishing, 2013.

[31] T. W. McKeithan, "Kinetic proofreading in T-cell receptor signal transduction," Proceedings of the National Academy of Sciences of the United States of America, vol. 92, no. 11, pp. 5042-5046, 1995.

[32] S. Valitutti, S. Muller, M. Cella, E. Padovan, and A. Lanzavecchia, "Serial triggering of many T-cell receptors by a few peptide-MHC complexes," Nature, vol. 375, no. 6527, pp. 148-151, 1995.

[33] S. Valitutti and A. Lanzavecchia, "Serial triggering of TCRs: a basis for the sensitivity and specificity of antigen recognition," Immunology Today, vol. 18, no. 6, pp. 299-304, 1997.

[34] E. San José, A. Borroto, F. Niedergang, A. Alcover, and B. Alarcón, "Triggering the TCR complex causes the downregulation of nonengaged receptors by a signal transductiondependent mechanism," Immunity, vol. 12, no. 2, pp. 161-170, 2000.

[35] C. Wofsy, D. Coombs, and B. Goldstein, "Calculations show substantial serial engagement of T cell receptors," Biophysical Journal, vol. 80, no. 2, pp. 606-612, 2001.
[36] M. Krogsgaard, Q. J. Li, C. Sumen, J. B. Huppa, M. Huse, and M. M. Davis, "Agonist/endogenous peptide-MHC heterodimers drive T cell activation and sensitivity," Nature, vol. 434, no. 7030, pp. 238-243, 2005.

[37] P. P. Yachi, J. Ampudia, N. R. J. Gascoigne, and T. Zal, "Nonstimulatory peptides contribute to antigen-induced CD8-T cell receptor interaction at the immunological synapse," Nature Immunology, vol. 6, no. 8, pp. 785-792, 2005.

[38] N. Anikeeva, D. Gakamsky, J. Scholler, and Y. Sykulev, "Evidence that the density of self peptide-MHC ligands regulates T-cell receptor signaling," PLoS One, vol. 7, no. 8, Article ID e41466, 2012.

[39] S. Tian, R. Maile, E. J. Collins, and J. A. Frelinger, " $C D^{8+} \mathrm{T}$ cell activation is governed by TCR-peptide/MHC affinity, not dissociation rate," Journal of Immunology, vol. 179, no. 5, pp. 29522960, 2007.

[40] J. D. Stone, A. S. Chervin, and D. M. Kranz, “T-cell receptor binding affinities and kinetics: impact on T-cell activity and specificity," Immunology, vol. 126, no. 2, pp. 165-176, 2009.

[41] O. Dushek, R. Das, and D. Coombs, "A role for rebinding in rapid and reliable T cell responses to antigen," PLoS Computational Biology, vol. 5, no. 11, Article ID e1000578, 2009.

[42] M. Aleksic, O. Dushek, H. Zhang et al., "Dependence of T cell antigen recognition on $\mathrm{T}$ cell receptor-peptide MHC confinement time," Immunity, vol. 32, no. 2, pp. 163-174, 2010.

[43] W. W. A. Schamel and M. Reth, "Monomeric and oligomeric complexes of the B cell antigen receptor," Immunity, vol. 13, no. 1, pp. 5-14, 2000.

[44] J. M. Dal Porto, S. B. Gauld, K. T. Merrell, D. Mills, A. E. PughBernard, and J. Cambier, "B cell antigen receptor signaling 101," Molecular Immunology, vol. 41, no. 6-7, pp. 599-613, 2004.

[45] C. Ulivieri and C. T. Baldari, “The BCR signalosome: where cell fate is decided," Journal of Biological Regulators and Homeostatic Agents, vol. 19, no. 1-2, pp. 1-16, 2005.

[46] P. Hou, E. Araujo, T. Zhao et al., "B cell antigen receptor signaling and internalization are mutually exclusive events," PLoS Biology, vol. 4, no. 7, p. e200, 2006.

[47] R. L. Geahlen, "Syk and pTyr'd: signaling through the B cell antigen receptor," Biochimica et Biophysica Acta, vol. 1793, no. 7, pp. 1115-1127, 2009.

[48] P. Tolar, H. W. Sohn, and S. K. Pierce, "Viewing the antigeninduced initiation of B-cell activation in living cells," Immunological Reviews, vol. 221, no. 1, pp. 64-76, 2008.

[49] S. K. Pierce and W. Liu, "The tipping points in the initiation of B cell signalling: how small changes make big differences," Nature Reviews Immunology, vol. 10, no. 11, pp. 767-777, 2010.

[50] N. E. Harwood and F. D. Batista, "Early events in B cell activation," Annual Review of Immunology, vol. 28, pp. 185-210, 2010.

[51] P. M. Waterman and J. C. Cambier, "The conundrum of inhibitory signaling by ITAM-containing immunoreceptors: potential molecular mechanisms," FEBS Letters, vol. 584, no. 24, pp. 4878-4882, 2010.

[52] M. Weber, B. Treanor, D. Depoil et al., "Phospholipase C- $\gamma 2$ and Vav cooperate within signaling microclusters to propagate B cell spreading in response to membrane-bound antigen," Journal of Experimental Medicine, vol. 205, no. 4, pp. 853-868, 2008.

[53] D. Depoil, S. Fleire, B. L. Treanor et al., "CD19 is essential for B cell activation by promoting $\mathrm{B}$ cell receptor-antigen microcluster formation in response to membrane-bound ligand," Nature Immunology, vol. 9, no. 1, pp. 63-72, 2008. 
[54] B. Treanor, D. Depoil, A. Bruckbauer, and F. D. Batista, "Dynamic cortical actin remodeling by ERM proteins controls BCR microcluster organization and integrity," Journal of Experimental Medicine, vol. 208, no. 5, pp. 1055-1068, 2011.

[55] P. Tolar, H. W. Sohn, and S. K. Pierce, “The initiation of antigeninduced B cell antigen receptor signaling viewed in living cells by fluorescence resonance energy transfer," Nature Immunology, vol. 6, no. 11, pp. 1168-1176, 2005.

[56] H. W. Sohn, P. Tolar, T. Jin, and S. K. Pierce, "Fluorescence resonance energy transfer in living cells reveals dynamic membrane changes in the initiation of B cell signaling," Proceedings of the National Academy of Sciences of the United States of America, vol. 103, no. 21, pp. 8143-8148, 2006.

[57] H. W. Sohn, S. K. Pierce, and S. J. Tzeng, "Live cell imaging reveals that the inhibitory Fc $\gamma$ RIIB destabilizes B cell receptor membrane-lipid interactions and blocks immune synapse formation," Journal of Immunology, vol. 180, no. 2, pp. 793-799, 2008.

[58] P. Tolar, J. Hanna, P. D. Krueger, and S. K. Pierce, “The constant region of the membrane immunoglobulin mediates B cellreceptor clustering and signaling in response to membrane antigens," Immunity, vol. 30, no. 1, pp. 44-55, 2009.

[59] S. P. Persaud, D. L. Donermeyer, K. S. Weber, D. M. Kranz, and P. M. Allen, "High-affinity T cell receptor differentiates cognate peptide-MHC and altered peptide ligands with distinct kinetics and thermodynamics," Molecular Immunology, vol. 47, no. 9, pp. 1793-1801, 2010.

[60] P. K. Tsourkas, N. Baumgarth, S. I. Simon, and S. Raychaudhuri, "Mechanisms of B-cell synapse formation predicted by Monte Carlo simulation," Biophysical Journal, vol. 92, no. 12, pp. 41964208, 2007.

[61] P. K. Tsourkas, M. L. Longo, and S. Raychaudhuri, "Monte Carlo study of single molecule diffusion can elucidate the mechanism of B cell synapse formation," Biophysical Journal, vol. 95, no. 3, pp. 1118-1125, 2008.

[62] A. S. Reddy, S. Chilukuri, and S. Raychaudhuri, "The network of receptors characterize B cell receptor micro- and macroclustering in a monte carlo model," Journal of Physical Chemistry B, vol. 114, no. 1, pp. 487-494, 2010.

[63] A. Srinivas Reddy, P. K. Tsourkas, and S. Raychaudhuri, "Monte Carlo study of B-cell receptor clustering mediated by antigen crosslinking and directed transport," Cellular and Molecular Immunology, vol. 8, no. 3, pp. 255-264, 2011.

[64] P. K. Tsourkas and S. Raychaudhuri, "Modeling of B cell synapse formation by monte carlo simulation shows that directed transport of receptor molecules is a potential formation mechanism," Cellular and Molecular Bioengineering, vol. 3, no. 3, pp. 256-268, 2010.

[65] P. K. Tsourkas and S. Raychaudhuri, "Monte carlo investigation of diffusion of receptors and ligands that bind across opposing surfaces," Annals of Biomedical Engineering, vol. 39, no. 1, pp. 427-442, 2011.

[66] K. Simons and R. Ehehalt, "Cholesterol, lipid rafts, and disease," Journal of Clinical Investigation, vol. 110, no. 5, pp. 597-603, 2002.

[67] K. Simons and D. Toomre, "Lipid rafts and signal transduction," Nature Reviews Molecular Cell Biology, vol. 1, no. 1, pp. 31-39, 2000.

[68] M. Triantafilou, K. Miyake, D. T. Golenbock, and K. Triantafilou, "Mediators of innate immune recognition of bacteria concentrate in lipid rafts and facilitate lipopolysaccharideinduced cell activation," Journal of Cell Science, vol. 115, part 12, pp. 2603-2611, 2002.

[69] V. Michel and M. Bakovic, "Lipid rafts in health and disease," Biology of the Cell, vol. 99, no. 3, pp. 129-140, 2007.

[70] R. Lindner and R. Knorr, "Rafting trips into the cell," Communitative and Integrative Biology, vol. 2, no. 5, pp. 420-421, 2009.

[71] S. Manes, G. del Real, and A. C. Martinez, "Pathogens: raft hijackers," Nature Reviews Immunology, vol. 3, no. 7, pp. 557568, 2003.

[72] P. C. Cheng, M. L. Dykstra, R. N. Mitchell, and S. K. Pierce, "A role for lipid rafts in B cell antigen receptor signaling and antigen targeting," Journal of Experimental Medicine, vol. 190, no. 11, pp. 1549-1560, 1999.

[73] M. J. Aman and K. S. Ravichandran, "A requirement for lipid rafts in B cell receptor induced $\mathrm{Ca}^{2+} \mathrm{x}$ flux," Current Biology, vol. 10, no. 7, pp. 393-396, 2000.

[74] M. Dykstra, A. Cherukuri, H. W. Sohn, S. J. Tzeng, and S. K. Pierce, "Location is everything: lipid rafts and immune cell signaling," Annual Review of Immunology, vol. 21, pp. 457-481, 2003.

[75] S. K. Pierce, "Lipid rafts and B-cell activation," Nature Reviews Immunology, vol. 2, no. 2, pp. 96-105, 2002.

[76] R. Xavier, T. Brennan, Q. Li, C. McCormack, and B. Seed, "Membrane compartmentation is required for efficient $\mathrm{T}$ cell activation," Immunity, vol. 8, no. 6, pp. 723-732, 1998.

[77] K. A. Field, D. Holowka, and B. Baird, "Compartmentalized activation of the high affinity immunoglobulin $\mathrm{E}$ receptor within membrane domains," Journal of Biological Chemistry, vol. 272, no. 7, pp. 4276-4280, 1997.

[78] K. A. Field, D. Holowka, and B. Baird, "Structural aspects of the association of FceRI with detergent-resistant membranes," Journal of Biological Chemistry, vol. 274, no. 3, pp. 1753-1758, 1999.

[79] A. Viola and N. Gupta, "Tether and trap: regulation of membrane-raft dynamics by actin-binding proteins," Nature Reviews Immunology, vol. 7, no. 11, pp. 889-896, 2007.

[80] H. W. Sohn, P. Tolar, and S. K. Pierce, "Membrane heterogeneities in the formation of B cell receptor-Lyn kinase microclusters and the immune synapse," Journal of Cell Biology, vol. 182, no. 2, pp. 367-379, 2008.

[81] N. Gupta and A. L. DeFranco, "Visualizing lipid raft dynamics and early signaling events during antigen receptor-mediated Blymphocyte activation," Molecular Biology of the Cell, vol. 14, no. 2, pp. 432-444, 2003.

[82] N. Gupta, B. Wollscheid, J. D. Watts, B. Scheer, R. Aebersold, and A. L. DeFranco, "Quantitative proteomic analysis of B cell lipid rafts reveals that ezrin regulates antigen receptor-mediated lipid raft dynamics," Nature Immunology, vol. 7, no. 6, pp. 625633, 2006.

[83] C. Klammt and B. F. Lillemeier, "How membrane structures control T cell signaling," Frontiers in Immunology, vol. 3, p. 291, 2012.

[84] W. W. Schamel and B. Alarcon, "Organization of the resting TCR in nanoscale oligomers," Immunological Reviews, vol. 251, no. 1, pp. 13-20, 2013.

[85] V. Horejsí, "Lipid rafts and their roles in T-cell activation," Microbes and Infection, vol. 7, no. 2, pp. 310-316, 2005.

[86] D. Filipp, O. Ballek, and J. Manning, "Lck, membrane microdomains, and TCR triggering machinery: defining the new rules of engagement," Frontiers in Immunology, vol. 3, p. 155, 2012. 
[87] D. A. Zacharias, J. D. Violin, A. C. Newton, and R. Y. Tsien, "Partitioning of lipid-modified monomeric GFPs into membrane microdomains of live cells," Science, vol. 296, no. 5569, pp. 913916, 2002.

[88] R. Varma and S. Mayor, "GPI-anchored proteins are organized in submicron domains at the cell surface," Nature, vol. 394, no. 6695, pp. 798-801, 1998.

[89] P. Sharma, R. Varma, R. C. Sarasij et al., "Nanoscale organization of multiple GPI-anchored proteins in living cell membranes," Cell, vol. 116, no. 4, pp. 577-589, 2004.

[90] D. E. Shvartsman, M. Kotler, R. D. Tall, M. G. Roth, and Y. I. Henis, "Differently anchored influenza hemagglutinin mutants display distinct interaction dynamics with mutual rafts," Journal of Cell Biology, vol. 163, no. 4, pp. 879-888, 2003.

[91] A. Pralle, P. Keller, E. L. Florin, K. Simons, and J. K. H. Hörber, "Sphingolipid-cholesterol rafts diffuse as small entities in the plasma membrane of mammalian cells," Journal of Cell Biology, vol. 148, no. 5, pp. 997-1008, 2000.

[92] W. K. Subczynski and A. Kusumi, "Dynamics of raft molecules in the cell and artificial membranes: approaches by pulse EPR spin labeling and single molecule optical microscopy," Biochimica et Biophysica Acta, vol. 1610, no. 2, pp. 231-243, 2003.

[93] K. Gaus, E. Gratton, E. P. W. Kable et al., "Visualizing lipid structure and raft domains in living cells with two-photon microscopy," Proceedings of the National Academy of Sciences of the United States of America, vol. 100, no. 26, pp. 15554-15559, 2003.

[94] D. M. Owen, D. Williamson, A. Magenau, and K. Gaus, “Optical techniques for imaging membrane domains in live cells (livecell palm of protein clustering)," Methods in Enzymology, vol. 504, pp. 221-235, 2012.

[95] T. M. Razzaq, P. Ozegbe, E. C. Jury, P. Sembi, N. M. Blackwell, and P. S. Kabouridis, "Regulation of T-cell receptor signaling by membrane microdomains," Immunology, vol. 113, no. 4, pp. 413426, 2004.

[96] M. Edidin, "The state of lipid rafts: from model membranes to cells," Annual Review of Biophysics and Biomolecular Structure, vol. 32, pp. 257-283, 2003.

[97] J. Liu, S. Qi, J. T. Groves, and A. K. Chakraborty, "Phase segregation on different length scales in a model cell membrane system," Journal of Physical Chemistry B, vol. 109, no. 42, pp. 19960-19969, 2005.

[98] G. Nudelman and Y. Louzoun, "Cell surface dynamics: the balance between diffusion, aggregation and endocytosis," IEE Proceedings Systems Biology, vol. 153, no. 1, pp. 34-42, 2006.

[99] G. Nudelman, M. Weigert, and Y. Louzoun, "In-silico cell surface modeling reveals mechanism for initial steps of B-cell receptor signal transduction," Molecular Immunology, vol. 46, no. 15, pp. 3141-3150, 2009.

[100] F. G. Karnell, R. J. Brezski, L. B. King, M. A. Silverman, and J. G. Monroe, "Membrane cholesterol content accounts for developmental differences in surface B cell receptor compartmentalization and signaling," Journal of Biological Chemistry, vol. 280, no. 27, pp. 25621-25628, 2005.

[101] M. E. J. Newman and G. T. Barkema, Monte Carlo Methods in Statistical Physics, Oxford University Press, New York, NY, USA, 1999.

[102] P. P. Yu-Yang, "Development of a Monte Carlo simulation of early events in B cell activation," in Biomedical Engineering, University of California, Davis, Calif, USA, 2012.
[103] F. Flores-Borja, P. S. Kabouridis, E. C. Jury, D. A. Isenberg, and R. A. Mageed, "Altered lipid raft-associated proximal signaling and translocation of CD45 tyrosine phosphatase in $\mathrm{B}$ lymphocytes from patients with systemic lupus erythematosus," Arthritis and Rheumatism, vol. 56, no. 1, pp. 291-302, 2007.

[104] V. R. Moulton and G. C. Tsokos, "Abnormalities of T cell signaling in systemic lupus erythematosus," Arthritis Research \& Therapy, vol. 13, no. 2, p. 207, 2011.

[105] M. Reth, "Oligomeric antigen receptors: a new view on signaling for the selection of lymphocytes," Trends in Immunology, vol. 22, no. 7, pp. 356-360, 2001.

[106] J. Yang and M. Reth, "Oligomeric organization of the B-cell antigen receptor on resting cells," Nature, vol. 467, no. 7314, pp. 465-469, 2010.

[107] A. Yethiraj and J. C. Weisshaar, "Why are lipid rafts not observed in vivo?” Biophysical Journal, vol. 93, no. 9, pp. 3113-3119, 2007.

[108] S. L. Veatch and S. L. Keller, "Miscibility phase diagrams of giant vesicles containing sphingomyelin," Physical Review Letters, vol. 94, no. 14, Article ID 148101, 2005.

[109] A. Jansson, "Kinetic proofreading and the search for nonselfpeptides," Self/Nonself, vol. 2, no. 1, pp. 1-3, 2011.

[110] J. Rossy, D. J. Williamson, C. Benzing, and K. Gaus, "The integration of signaling and the spatial organization of the $\mathrm{T}$ cell synapse," Frontiers in Immunology, vol. 3, p. 352, 2012.

[111] J. R. James, J. McColl, M. I. Oliveira, P. D. Dunne, E. Huang, A. Jansson et al., "The $\mathrm{T}$ cell receptor triggering apparatus is composed of monovalent or monomeric proteins," The Journal of Biological Chemistry, vol. 286, no. 37, pp. 31993-32001, 2011.

[112] R. N. Germain, "Computational analysis of T cell receptor signaling and ligand discrimination-past, present, and future," FEBS Letters, vol. 584, no. 24, pp. 4814-4822, 2010.

[113] T. Mamo and G. A. Poland, "Nanovaccinology: the next generation of vaccines meets 21st century materials science and engineering," Vaccine, vol. 30, no. 47, pp. 6609-6611, 2012.

[114] S. Y. Qi, J. T. Groves, and A. K. Chakraborty, "Synaptic pattern formation during cellular recognition," Proceedings of the National Academy of Sciences of the United States of America, vol. 98, no. 12, pp. 6548-6553, 2001.

[115] N. J. Burroughs and C. Wülfing, "Differential segregation in a cell-cell contact interface: the dynamics of the immunological synapse," Biophysical Journal, vol. 83, no. 4, pp. 1784-1796, 2002.

[116] S. Raychaudhuri, A. K. Chakraborty, and M. Kardar, "Effective membrane model of the immunological synapse," Physical Review Letters, vol. 91, no. 20, Article ID 208101, 4 pages, 2003.

[117] T. R. Weikl and R. Lipowsky, "Pattern formation during T-cell adhesion," Biophysical Journal, vol. 87, no. 6, pp. 3665-3678, 2004.

[118] D. Coombs, M. Dembo, C. Wofsy, and B. Goldstein, "Equilibrium thermodynamics of cell-cell adhesion mediated by multiple ligand-receptor Pairs," Biophysical Journal, vol. 86, no. 3, pp. 1408-1423, 2004.

[119] K. I. Lim and J. Yin, "Localization of receptors in lipid rafts can inhibit signal transduction," Biotechnology and Bioengineering, vol. 90, no. 6, pp. 694-702, 2005.

[120] K. H. Lee, A. R. Dinner, C. Tu et al., "The immunological synapse balances $\mathrm{T}$ cell receptor signaling and degradation," Science, vol. 302, no. 5648, pp. 1218-1222, 2003.

[121] C. R. F. Monks, B. A. Freiberg, H. Kupfer, N. Sciaky, and A. Kupfer, "Three-dimensional segregation of supramolecular activation clusters in T cells," Nature, vol. 395, no. 6697, pp. 82$86,1998$. 
[122] A. Grakoui, S. K. Bromley, C. Sumen et al., "The immunological synapse: a molecular machine controlling T cell activation," Science, vol. 285, no. 5425, pp. 221-227, 1999.

[123] F. D. Batista, D. Iber, and M. S. Neuberger, "B cells acquire antigen from target cells after synapse formation," Nature, vol. 411, no. 6836, pp. 489-494, 2001.

[124] Y. R. Carrasco, S. J. Fleire, T. Cameron, M. L. Dustin, and F. D. Batista, "LFA-1/ICAM-1 interaction lowers the threshold of B cell activation by facilitating $B$ cell adhesion and synapse formation," Immunity, vol. 20, no. 5, pp. 589-599, 2004.

[125] S. Čemerski, J. Das, E. Giurisato et al., "The balance between $\mathrm{T}$ cell receptor signaling and degradation at the center of the immunological synapse is determined by antigen quality," Immunity, vol. 29, no. 3, pp. 414-422, 2008.

[126] D. H. Busch and E. G. Pamer, "T cell affinity maturation by selective expansion during infection," Journal of Experimental Medicine, vol. 189, no. 4, pp. 701-709, 1999.

[127] P. A. Savage, J. J. Boniface, and M. M. Davis, "A kinetic basis for T cell receptor repertoire selection during an immune response," Immunity, vol. 10, no. 4, pp. 485-492, 1999.

[128] A. Amranl, J. Verdaguer, P. Serra, S. Tafuro, R. Tan, and P. Santamaria, "Progression of autoimmune diabetes driven by avidity maturation of a T- cell population," Nature, vol. 406, no. 6797, pp. 739-742, 2000.

[129] M. K. Slifka and J. L. Whitton, "Functional avidity maturation of $\mathrm{CD}^{8+} \mathrm{T}$ cells without selection of higher affinity TCR," Nature Immunology, vol. 2, no. 8, pp. 711-717, 2001.

[130] A. S. Perelson, M. Mirmirani, and G. F. Oster, "Optimal strategies in immunology. I. B cell differentiation and proliferation," Journal of Mathematical Biology, vol. 3, no. 3-4, pp. 325-367, 1976.

[131] A. S. Perelson, M. Mirmirani, and G. F. Oster, "Optimal strategies in immunology. II. B memory cell production," Journal of Mathematical Biology, vol. 5, no. 3, pp. 213-256, 1978.

[132] A. S. Perelson and G. F. Oster, "Theoretical studies of clonal selection: minimal antibody repertoire size and reliability of self-non-self discrimination," Journal of Theoretical Biology, vol. 81, no. 4, pp. 645-670, 1979.

[133] Z. Agur, G. Mazor, and I. Meilijson, "Maturation of the humoral immune response as an optimization problem," Proceedings of the Royal Society, vol. 245, no. 1313, pp. 147-150, 1991.

[134] T. B. Kepler and A. S. Perelson, "Somatic hypermutation in B cells: an optimal control treatment," Journal of Theoretical Biology, vol. 164, no. 1, pp. 37-64, 1993.

[135] H. Qi, J. G. Egen, A. Y. C. Huang, and R. N. Germain, "Extrafollicular activation of lymph node B cells by antigenbearing dendritic cells," Science, vol. 312, no. 5780, pp. 16721676, 2006.

[136] C. D. C. Allen, T. Okada, H. L. Tang, and J. G. Cyster, "Imaging of germinal center selection events during affinity maturation," Science, vol. 315, no. 5811, pp. 528-531, 2007.

[137] Y. R. Carrasco and F. D. Batista, "B cells acquire particulate antigen in a macrophage-rich area at the boundary between the follicle and the subcapsular sinus of the lymph node," Immunity, vol. 27, no. 1, pp. 160-171, 2007.

[138] T. Junt, E. A. Moseman, M. Iannacone et al., "Subcapsular sinus macrophages in lymph nodes clear lymph-borne viruses and present them to antiviral B cells," Nature, vol. 450, no. 7166, pp. 110-114, 2007.

[139] T. G. Phan, I. Grigorova, T. Okada, and J. G. Cyster, "Subcapsular encounter and complement-dependent transport of immune complexes by lymph node B cells," Nature Immunology, vol. 8, no. 9, pp. 992-1000, 2007.

[140] F. D. Batista and N. E. Harwood, "The who, how and where of antigen presentation to B cells," Nature Reviews Immunology, vol. 9, no. 1, pp. 15-27, 2009.

[141] M. Meyer-Hermann, M. T. Figge, and K. M. Toellner, “Germinal centres seen through the mathematical eye: B-cell models on the catwalk," Trends in Immunology, vol. 30, no. 4, pp. 157-164, 2009.

[142] H. P. Mirsky, M. J. Miller, J. J. Linderman, and D. E. Kirschner, "Systems biology approaches for understanding cellular mechanisms of immunity in lymph nodes during infection," Journal of Theoretical Biology, vol. 287, pp. 160-170, 2011.

[143] R. M. Vroomans, A. F. Maree, R. J. de Boer, and J. B. Beltman, "Chemotactic migration of $\mathrm{T}$ cells towards dendritic cells promotes the detection of rare antigens," PLOS Computational Biology, vol. 8, no. 11, Article ID e1002763, 2012.

[144] M. T. Figge, A. Garin, M. Gunzer, M. Kosco-Vilbois, K. M. Toellner, and M. Meyer-Hermann, "Deriving a germinal center lymphocyte migration model from two-photon data," Journal of Experimental Medicine, vol. 205, no. 13, pp. 3019-3029, 2008.

[145] Y. Takahashi, D. M. Cerasoli, J. M. Dal Porto et al., "Relaxed negative selection in germinal centers and impaired affinity maturation in bcl-x(L) transgenic mice," Journal of Experimental Medicine, vol. 190, no. 3, pp. 399-409, 1999.

[146] S. Raychaudhuri, E. Willgohs, T. N. Nguyen, E. M. Khan, and T. Goldkorn, "Monte Carlo simulation of cell death signaling predicts large cell-to-cell stochastic fluctuations through the type 2 pathway of apoptosis," Biophysical Journal, vol. 95, no. 8, pp. 3559-3562, 2008.

[147] J. G. Albeck, J. M. Burke, S. L. Spencer, D. A. Lauffenburger, and P. K. Sorger, "Modeling a snap-action, variable-delay switch controlling extrinsic cell death," PLoS Biology, vol. 6, no. 12, article e299, pp. 2831-2852, 2008.

[148] H. Düssmann, M. Rehm, C. G. Concannon et al., "Single-cell quantification of Bax activation and mathematical modelling suggest pore formation on minimal mitochondrial Bax accumulation," Cell Death and Differentiation, vol. 17, no. 2, pp. 278290, 2010.

[149] J. Skommer, T. Brittain, and S. Raychaudhuri, "Bcl-2 inhibits apoptosis by increasing the time-to-death and intrinsic cellto-cell variations in the mitochondrial pathway of cell death," Apoptosis, vol. 15, no. 10, pp. 1223-1233, 2010.

[150] J. Skommer, S. C. Das, A. Nair, T. Brittain, and S. Raychaudhuri, "Nonlinear regulation of commitment to apoptosis by simultaneous inhibition of $\mathrm{Bcl}-2$ and XIAP in leukemia and lymphoma cells," Apoptosis, vol. 16, no. 6, pp. 619-626, 2011.

[151] S. Raychaudhuri, "How can we kill cancer cells: insights from the computational models of apoptosis," World Journal of Clinical Oncology, vol. 1, no. 1, pp. 24-28, 2010.

[152] P. S. Kabouridis and E. C. Jury, "Lipid rafts and T-lymphocyte function: implications for autoimmunity," FEBS Letters, vol. 582, no. 27, pp. 3711-3718, 2008.

[153] J. Surls, C. Nazarov-Stoica, M. Kehl, C. Olsen, S. Casares, and T. D. Brumeanu, "Increased membrane cholesterol in lymphocytes diverts T-cells toward an inflammatory response," PLoS One, vol. 7, no. 6, Article ID e38733, 2012.

[154] A. K. Dey and I. K. Srivastava, "Novel adjuvants and delivery systems for enhancing immune responses induced by immunogens," Expert Review of Vaccines, vol. 10, no. 2, pp. 227-251, 2011. 


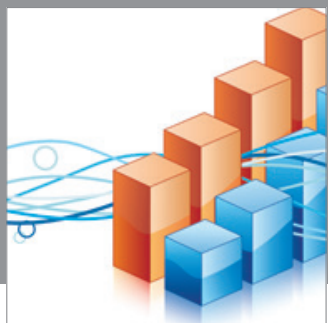

Advances in

Operations Research

mansans

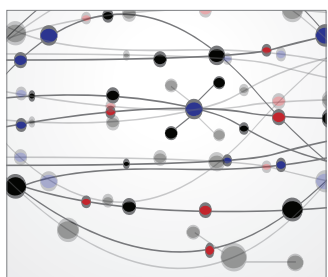

The Scientific World Journal
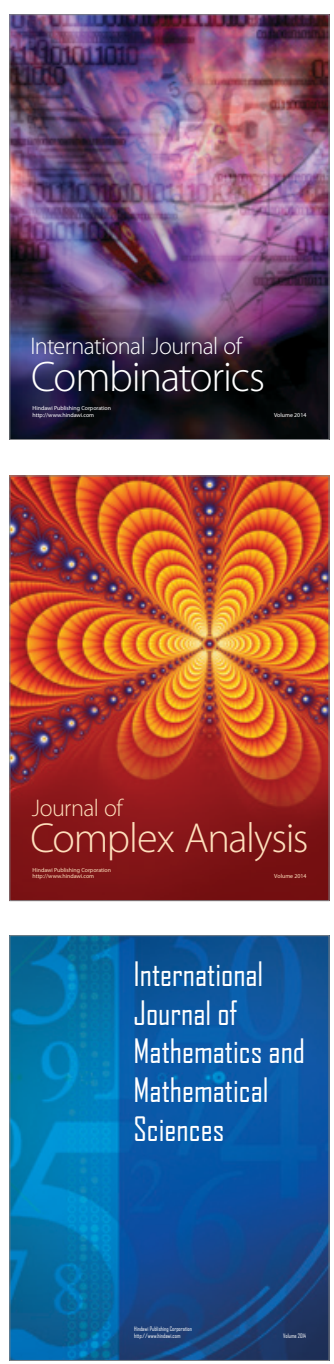
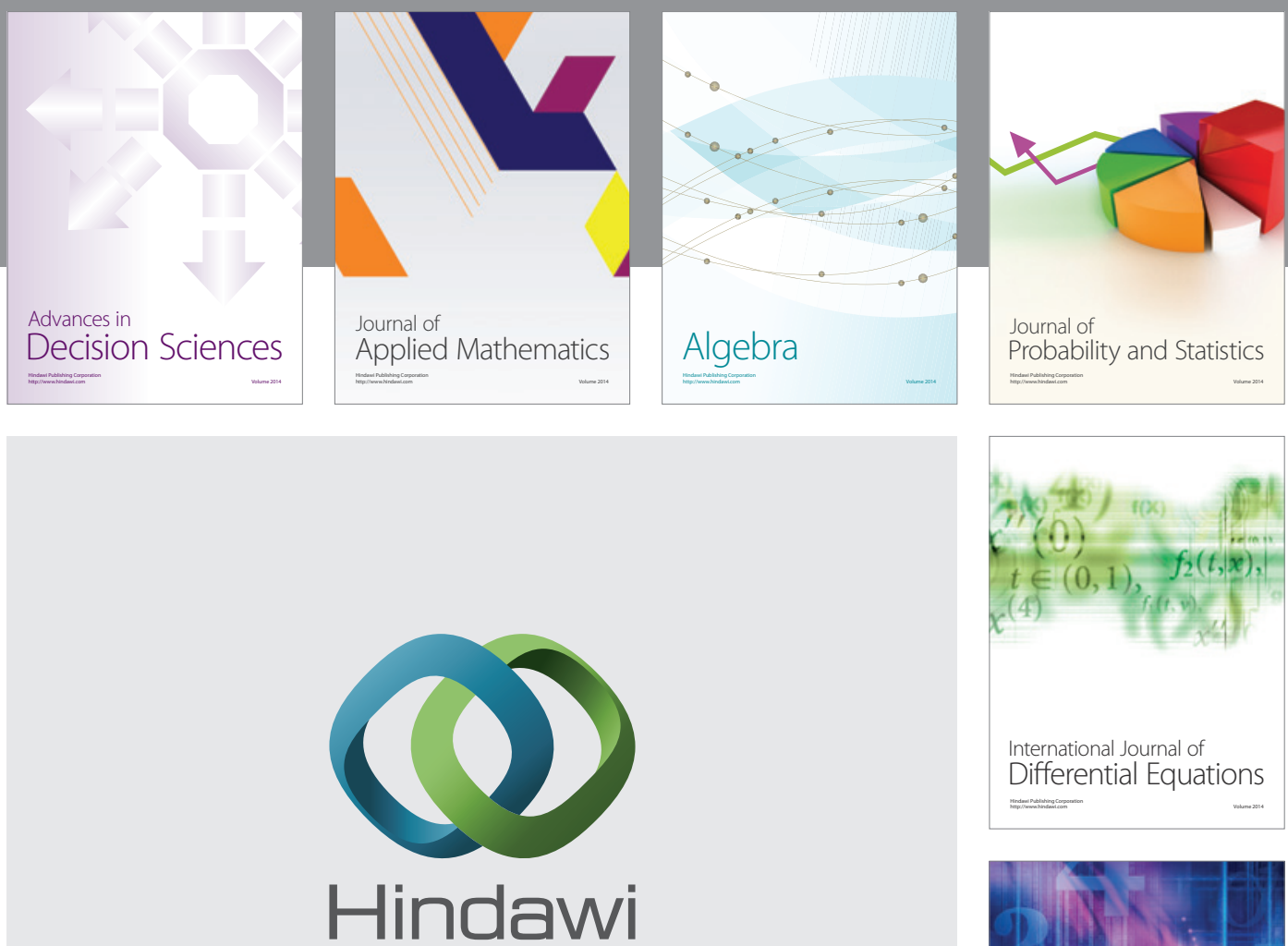

Submit your manuscripts at http://www.hindawi.com
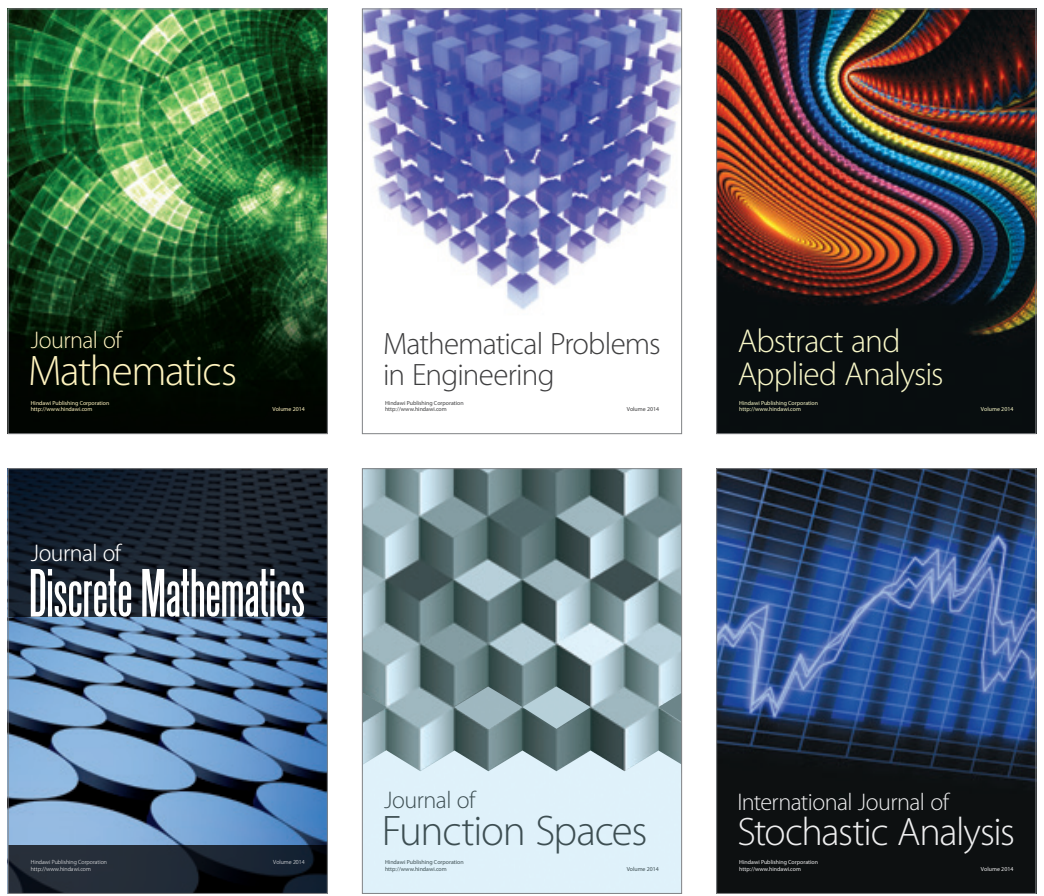

Journal of

Function Spaces

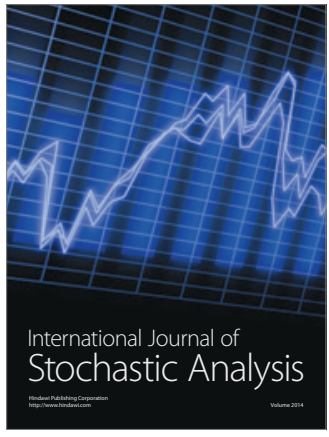

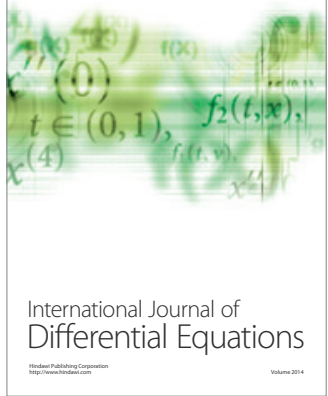
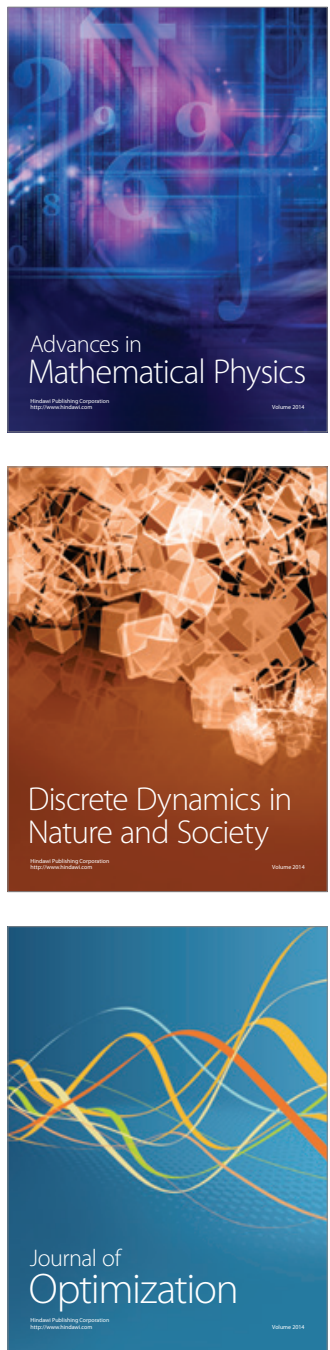